Rev. Int. Contam. Ambie. 35 (4) 869-888, 2019

DOI: 10.20937/RICA.2019.35.04.08

\title{
ANÁLISIS DEL TRANSPORTE DE OZONO EN LA CUENCA ATMOSFÉRICA DE PUEBLA-TLAXCALA EN EL CENTRO DE MÉXICO
}

\author{
Analysis of ozone transport in the atmospheric basin of Puebla-Tlaxcala in central Mexico
}

\begin{abstract}
Hugo Alberto BARRERA HUERTAS ${ }^{1 *}$, Ricardo TORRES JARDÓN ${ }^{1}$, Luis Gerardo RUÍZ SUÁREZ ${ }^{1}$, José Santos GARCÍA YEE ${ }^{1}$, Alejandro TORRES JARAMILLO ${ }^{2}$, Ana Patricia MARTÍNEZ BOLÍlVAR ${ }^{3}$, Wilfrido GUTIÉRREZ LÓPEZ ${ }^{1}$, León Manuel GARCÍA Y ESPINOSA DE LOS REYES ${ }^{1}$, Miguel ROBLES ${ }^{1}$, Armando RETAMA HERNANDEZ ${ }^{4}$ y José Agustín GARCÍA REYNOSO ${ }^{1}$
\end{abstract}

${ }^{1}$ Centro de Ciencias de la Atmósfera, Universidad Nacional Autónoma de México, Avenida Universidad 3000, Universidad Nacional Autónoma de México CU, Ciudad de México C.P. 04510, México

${ }^{2}$ Departamento de Investigación en Zeolitas, Benemérita Universidad Autónoma de Puebla, Edificio IC12 Ciudad Universitaria, Puebla-Puebla, C.P. 72570

${ }^{3}$ Gestión de la Calidad del Aire y Registro de Emisiones y Transferencia de Contaminantes, Secretaría del Medio Ambiente y Recursos Naturales, Av. Ejército Nacional 223, Col. Anáhuac, Ciudad de México. C.P. 11320, México

${ }^{4}$ Dirección de Monitoreo Atmosférico, Secretaría del Medio Ambiente, Gran Avenida Colonia Lomas de Chapultepec, Sección I, Delegación Miguel Hidalgo, Ciudad de México, C.P. 11000

*Autor de correspondencia: hbarrera@atmosfera.unam.mx

(Recibido: septiembre 2017, aceptado: noviembre 2018)

Palabras clave: contaminantes atmosféricos, dinámica de ozono, intercambio intercuencas, ozonosondeos

\section{RESUMEN}

Se estudió la dinámica del transporte y ocurrencia de ozono en la cuenca atmosférica del valle de Puebla-Tlaxcala (VPT) durante marzo y abril de 2012. Se monitorearon ozono $\left(\mathrm{O}_{3}\right)$, óxidos de nitrógeno (NOx), y variables meteorológicas en superficie en los sitios rurales Huaquechula (HUAQ), Chipilo (CHIP), y Amozoc (AMOZ), y se midió intermitentemente el perfil vertical de $\mathrm{O}_{3}$ y las condiciones meteorológicas asociadas en CHIP. La norma de calidad del aire mexicana para $\mathrm{O}_{3}$ de 1 hora de $0.095 \mathrm{ppm}$, se rebasó en CHIP y AMOZ en cinco y una ocasión respectivamente. Se observó un incremento matutino rápido de $\mathrm{O}_{3}$ en los tres sitios asociado al mezclado de $\mathrm{O}_{3}$ por arriba de la capa de inversión conforme se desarrolló la capa de mezcla. Se registraron eventos vespertinos inusuales de $\mathrm{O}_{3}$ alrededor de $0.080 \mathrm{ppm}$ sostenidos por varias horas en los tres sitios que podrían estar asociados a la recirculación de masas de aire dentro del VPT y el $\mathrm{O}_{3}$ transportado advectivamente del valle Cuernavaca-Cuautla. El perfil vertical de $\mathrm{O}_{3}$ mostró un incremento con la altura a concentraciones mayores que las observadas en superficie y el análisis de trayectorias de retroceso en 2-D sugirió condiciones favorables al acarreo de $\mathrm{O}_{3}$ de la troposfera libre hacia la superficie. Se concluye que el establecimiento de estrategias de control en el VPT debe considerar la influencia del intercambio de $\mathrm{O}_{3}$ entre cuencas, la estacionalidad meteorológica local y sinóptica, y las posibles contribuciones de $\mathrm{O}_{3}$ de la troposfera libre y no sólo limitarse a las condiciones locales.

Key words: ozone dynamics, atmospheric pollutants, inter-basin interchange, ozonesounding 


\begin{abstract}
A study on the dynamic of transport and occurrence of ozone in the air basin of the Puebla-Tlaxcala Valley (PTV) was performed between March and April 2012. The ozone $\left(\mathrm{O}_{3}\right)$, nitrogen oxides $(\mathrm{NOx})$ and several meteorological variables were measured at the surface level in the rural sites of Huaquechula (HUAQ), Chipilo (CHIP), and Amozoc (AMOZ). The vertical $\mathrm{O}_{3}$ profile and the associated meteorological conditions, were measured intermittently in CHIP. The 1 hour Mexican air quality standard of $0.095 \mathrm{ppm}$ for $\mathrm{O}_{3}$, was exceeded in five and one days in CHIP and $\mathrm{AMOZ}$, respectively. A rapid increase in the morning for $\mathrm{O}_{3}$ levels was observed in the three sites and was associated with the mixing of $\mathrm{O}_{3}$ above the inversion layer once started the growing of the turbulent mixing layer. Unusual $\mathrm{O}_{3}$ evening events of around $0.080 \mathrm{ppm}$ were recorded for several hours in the three sites that could be associated with the recirculation of air masses with ozone formed within the VPT and $\mathrm{O}_{3}$ advectively transported from the Cuernavaca-Cuautla Valley. The vertical $\mathrm{O}_{3}$ profile showed an increase with height at concentrations even higher than those observed at the surface, and the 2-D analysis of backward trajectories suggested favorable conditions for $\mathrm{O}_{3}$ transported from the free troposphere to the surface. It is concluded that the implementation of control strategies in the VPT should consider the influence of the inter-basin exchange, the meteorological local and synoptic seasonality, and the possible contributions of $\mathrm{O}_{3}$ from the free troposphere and not be limited to local conditions.
\end{abstract}

\section{INTRODUCCIÓN}

El ozono $\left(\mathrm{O}_{3}\right)$ en la tropósfera baja es un contaminante secundario formado a partir de una compleja serie de reacciones entre los óxidos de nitrógeno $\left(\mathrm{NO}_{\mathrm{x}}=\mathrm{NO}+\mathrm{NO}_{2}\right)$ y los compuestos orgánicos volátiles (COV) en presencia de luz solar y cuyo control no depende linealmente de la reducción indiscriminada de sus precursores (Finlayson-Pitts y Pitts 2000). La importancia de las concentraciones de $\mathrm{O}_{3}$ registradas viento abajo de zonas urbanas radica en que frecuentemente son mayores a aquellas zonas emisoras de sus precursores, teniendo efectos negativos en la salud de la población y la vegetación en zonas rurales y forestales bajo influencia de la pluma de contaminación urbana (Krupa 1999).

El problema del $\mathrm{O}_{3}$ ha sido ampliamente documentado para la Cuenca del Valle de México en donde se ha identificado la ocurrencia de un intercambio de masas de aire contaminado entre la cuenca atmosférica donde se asienta la zona urbana de la Ciudad de México y las cuencas atmosféricas vecinas (de Foy et al. 2005, Jazcilevich et al. 2005, Molina et al. 2007, INE 2009, García-Reynoso 2009, Molina et al. 2010, Salcedo et al. 2012). Salcedo et al. (2012) investigaron la dinámica de la contaminación del aire en la cuenca Cuernavaca-Cuautla (CCC) y encuentran que, en ocasiones, es posible el transporte de masas de aire con $\mathrm{O}_{3}$ entre esta cuenca y la del valle de Puebla-Tlaxcala (VPT) a través de la microrregión Cuautla-Atlixco. Sin embargo, a pesar de su importancia en la influencia de este transporte en la calidad del aire de Puebla no existe más información sobre esta posibilidad.

Desde la perspectiva del diseño de política ambiental en México, se reconoce una gran complejidad en el control eficaz de la calidad del aire en las diferentes regiones del centro de México debido a la influencia que su compleja orografía tiene sobre los vientos de superficie y su transporte atmosférico entre las diferentes cuencas atmosféricas que 1o componen (CAMe 2017). Una cuenca atmosférica se define como "un espacio geográfico delimitado parcial o totalmente por elevaciones montañosas $u$ otros atributos naturales con características meteorológicas y climáticas afines, donde la calidad del aire a nivel estacional está influenciada por las fuentes de emisión antropogénicas y naturales en el interior de esta, y por concentraciones de fondo que llegan a la cuenca" (INE 2007).

La importancia de este intercambio entre cuencas alcanza otra dimensión al reconocerse la magnitud de la megalópolis del centro del país. Esta macroregión está conformada por la zonas Metropolitana de la Ciudad de México (ZMCM), del valle de Puebla-Tlaxcala (ZMVPT), la zona metropolitana Toluca-Lerma (ZMTL) y las áreas conurbadas de Cuernavaca-Jiutepec, Cuautla y Pachuca (Fig. 1), 


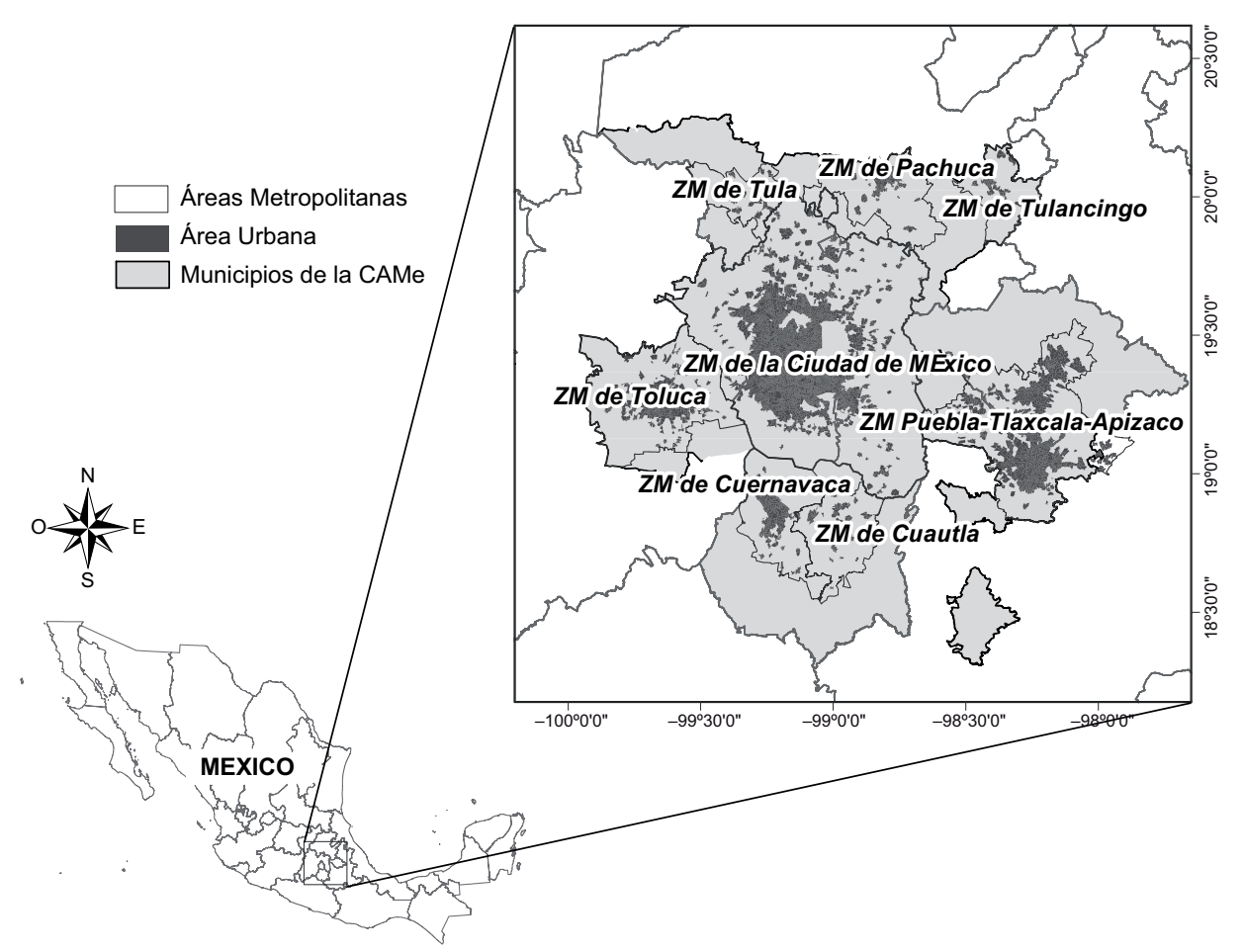

Fig. 1. Ubicación geográfica de la megalópolis del centro de México, estados, ciudades y áreas metropolitanas que la conforman (Modificado del Consejo Estatal de Población, Hidalgo 2001)

con una población aproximada de 27 millones de habitantes (CAMe 2017). La región geográfica que cubre esta megalópolis se localiza en el Altiplano Mexicano por arriba de los $1500 \mathrm{msnm}$, alcanzando elevaciones mayores a los $4500 \mathrm{msnm}$ y es influenciada por sistemas meteorológicos sinópticos tropicales y de latitudes medias.

Sin embargo, salvo la ZMCM que ha sostenido la operación de una red de monitoreo de calidad del aire para su vigilancia, hay todavía muchas deficiencias en las redes de los estados vecinos que han limitado tanto la evaluación de la tendencia en los niveles locales como el entendimiento de la dinámica de transporte del $\mathrm{O}_{3}$ entre las diversas cuencas y, en consecuencia, su influencia en la calidad del aire local y regional. Como resultado se han generado diferentes programas de mejoramiento de la calidad del aire enfocados al problema aislado a cada zona metropolitana cuyos resultados no indican un control en la problemática.

El objetivo de este trabajo es presentar resultados de un análisis de la dinámica del transporte de $\mathrm{O}_{3}$ entre las cuencas atmosféricas vecinas y la influencia de otras contribuciones atmosféricas sobre la calidad del aire del VPT.

\section{MATERIALES Y MÉTODOS}

\section{Descripción de la zona de estudio}

La ZMVPT es la región urbana resultante de la conurbación de varios municipios del centro del estado de Puebla y del sur de Tlaxcala. Se ubica entre los paralelos $18^{\circ} 50^{\prime}$ y $19^{\circ} 14^{\prime}$ de latitud norte y los meridianos $98^{\circ} 01^{\prime}$ y $98^{\circ} 18^{\prime}$ ' de longitud oeste, ocupando una superficie de $2392.4 \mathrm{~km}^{2}$ y cuenta con una población estimada de 2728790 habitantes (INEGI 2010). El VPT se ubica a una altitud aproximada de $2160 \mathrm{msnm}$; al norte extiende su superficie dentro del estado de Tlaxcala, hacia el oeste y noroeste limitan con la Sierra Nevada formada por los volcanes Popocatépetl e Iztaccíhuatl, que alcanzan altitudes superiores a los $5000 \mathrm{msnm}$; al noreste se encuentra con el volcán Malinche con una altitud de 4420 msnm, y por el sur se extiende por el resto de la zona sur de Puebla (Fig. 2). Por su parte, Cuautla se localiza en la zona oriente del estado de Morelos entre los paralelos $18^{\circ} 45^{\prime}$ y $18^{\circ} 53^{\prime}$ de latitud norte y los meridianos $98^{\circ}$ $53^{\prime}$ y $99^{\circ} 01^{\prime}$ de longitud oeste y se ubica al sur de la Sierra Chichinautzin y al suroeste de la Sierra Nevada en una planicie de elevación relativamente constante teniendo hacia el sur una cadena de montañas. El 


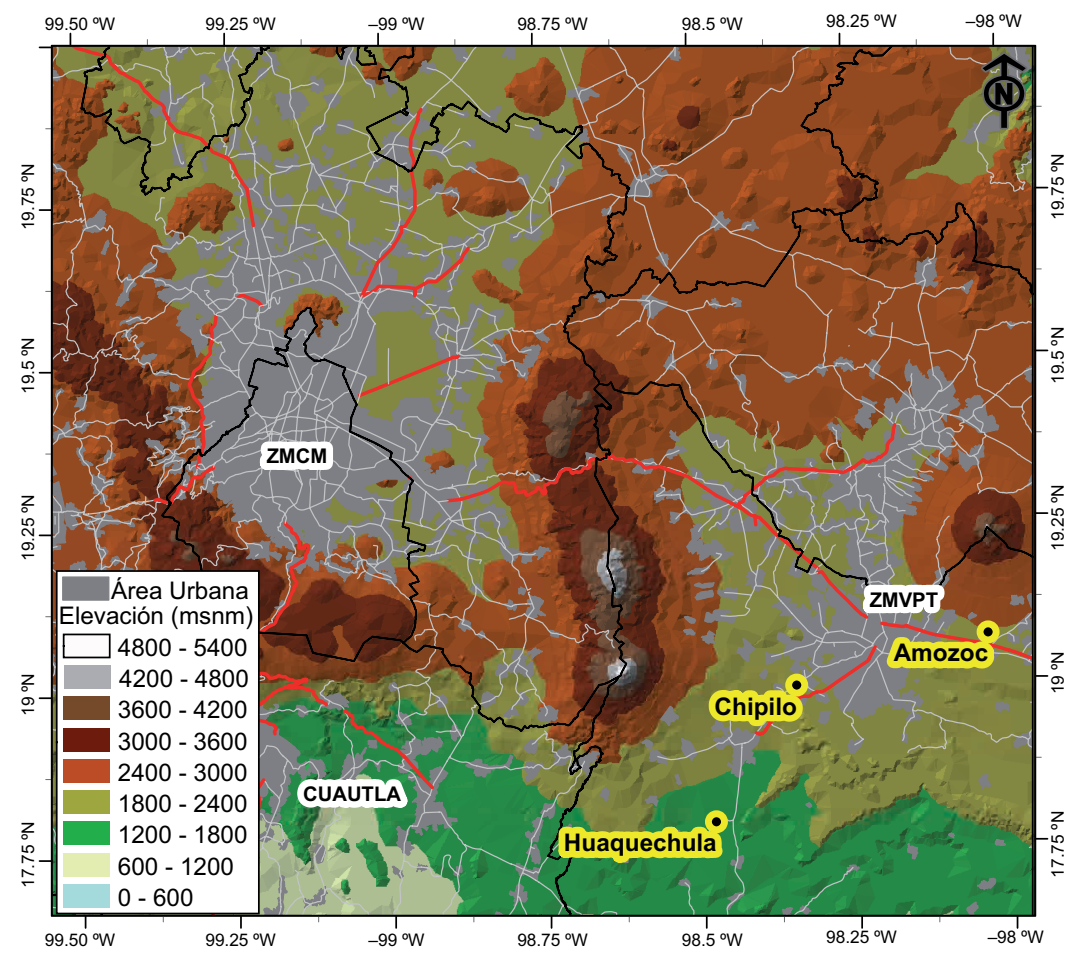

Fig. 2. Localización de los sitios de medición en la Zona Metropolitana del Valle de Puebla-Tlaxcala. Los contornos negros indican los límites estatales, los contornos rojos denotan vías principales de traslado como autopistas

valle de Cuautla se ubica a una altitud aproximada de $1330 \mathrm{msnm}$ y ocupa una superficie de $153651 \mathrm{~km}^{2}$ con una población estimada de 175207 habitantes (INEGI 2010). Ambas regiones se comunican a través de un paso de montaña relativamente amplio ubicado al suroeste del VPT.

De acuerdo con la clasificación climática de Köppen, el VPT posee un clima templado $(\mathrm{Cw})$, que conforme se avanza hacia el sur y disminuye su altitud cambia a cálido subhúmedo $\mathrm{A}(\mathrm{Cw} 1)$, que es también el clima que predomina en el valle de Cuautla con lluvias en los meses de junio a octubre. Los patrones de circulación de los vientos en esta región son dominados por la circulación de valle-montaña (Lauer y Klaus 1975). Durante el día, en términos generales hay una circulación norte a sur por la mañana, y hasta mediodía que, conforme avanza el calentamiento del día, va cambiando a una dominancia vespertina de vientos del sur y suroeste promovidos por corrientes que ingresan por el Océano Pacífico. Por la noche se presentan vientos dominantes del sector norte del VPT influenciados por escurrimientos de vientos de la Malinche y de la Sierra Nevada, mientras que en Cuautla influyen escurrimientos del noroeste y noreste de la Sierra Chichinautzin y del Popocatépetl (Jáuregui 1968, Vidal 1980).

\section{Campaña de mediciones}

La campaña de mediciones Puebla-Tlaxcala 2012 corresponde a la temporada cálida seca, cuando la radiación solar es intensa e incluye parte de la temporada de $\mathrm{O}_{3}$ en el centro de México. Se ubicaron tres unidades móviles equipadas con instrumentos para la determinación de calidad del aire y parámetros meteorológicos; una de ellas perteneciente al Instituto Nacional de Ecología y Cambio Climático (INECC) montada en Amozoc (AMOZ) y otras dos pertenecientes al Centro de Ciencias de la Atmósfera de la Universidad Nacional Autónoma de México (CCA-UNAM) montadas en Chipilo (CHIP) y Huaquechula (HUAQ) (Fig. 2). El cuadro I describe los instrumentos utilizados en cada uno de los sitios con mediciones en superficie y con el globo cautivo instrumentado. Las mediciones del perfil vertical de $\mathrm{O}_{3}$ y de las variables meteorológicas se efectuaron acoplando una ozonosonda con instrumentación meteorológica a un globo cautivo sólo en el sitio CHIP. La medición del perfil de $\mathrm{O}_{3}$ se realizó con una ozonosonda conformada por una celda electroquímica (redox iodo-iodo) en las fechas y horas que se muestran en el cuadro II. Se programaron tres sondeos al día (09:00 h, 12:00 h y 18:00 h) con la intención de conocer la evolución de la capa de mezcla diurna y su relación con las 
CUADRO I. EQUIPAMIENTO DE LAS UNIDADES DE MONITOREO ATMOSFÉRICO DURANTE LA CAMPAÑA DE MUESTREO (30 MARZO AL 27 ABRIL DEL 2012)

\begin{tabular}{|c|c|c|c|c|}
\hline Parámetro & Principio & Tecnología/ Modelo & Precisión & Límite detectable \\
\hline \multirow{3}{*}{$\mathrm{O}_{3}$} & \multirow{3}{*}{$\begin{array}{l}\text { Fotometría de absorción } \\
\text { ultravioleta }\end{array}$} & $\begin{array}{l}\text { Advanced Pollution Instruments } \\
400 \text { Modelo } 1006\end{array}$ & $0.5 \mathrm{ppb}$ & $0.4 \mathrm{ppb}$ \\
\hline & & $\begin{array}{l}\text { Thermo Environmental Instruments } \\
\text { Modelo 49C }\end{array}$ & $1.0 \mathrm{ppb}$ & $1.0 \mathrm{ppb}$ \\
\hline & & $\begin{array}{l}\text { Integrated Air-pointer } \\
\text { multi-gas measurement platform } \\
\text { (Recordum Messtechnik GmbH) }\end{array}$ & $1.0 \mathrm{ppb}$ & $20 \mathrm{ppb}$ \\
\hline \multirow[b]{2}{*}{$\mathrm{NO}, \mathrm{NO}_{2}$} & \multirow{2}{*}{$\begin{array}{l}\text { Quimioluminiscencia. } \\
\text { Convertidor interno de } \\
\text { Molibdeno }\end{array}$} & $\begin{array}{l}\text { Thermo Environmental Instruments } \\
\text { Modelo } 42 \mathrm{C}\end{array}$ & $\pm 0.4 \mathrm{ppb}$ & $0.40 \mathrm{ppb}$ \\
\hline & & $\begin{array}{l}\text { Integrated Air-pointer } \\
\text { multi-gas measurement platform } \\
\text { (Recordum Messtechnik GmbH) }\end{array}$ & $1.0 \mathrm{ppb}$ & $\begin{array}{l}\text { Dinámico } \\
\text { hasta } 20 \mathrm{ppm}\end{array}$ \\
\hline \multirow{3}{*}{ Temperatura } & \multirow{3}{*}{ Resistencia de platino } & Vaisala. Modelo HMP45A & $\pm 0.2^{\circ} \mathrm{C}\left(20^{\circ} \mathrm{C}\right)$ & $-32.9^{\circ}-+60^{\circ} \mathrm{C}$ \\
\hline & & Vaisala. WXT520 AAB0BC10B0 & $\pm 0.3^{\circ} \mathrm{C}\left(20^{\circ} \mathrm{C}\right)$ & $-52^{\circ}-+60^{\circ} \mathrm{C}$ \\
\hline & & Met One. Modelo 062 & $\pm 0.1^{\circ} \mathrm{C}$ & $-50^{\circ}-+50^{\circ} \mathrm{C}$ \\
\hline \multirow{3}{*}{ Humedad relativa } & \multirow{3}{*}{ Sensor capacitivo } & Vaisala. Modelo HMP45A & $\pm 1 \% \mathrm{RH}$ & $\begin{array}{l}0.8-100 \% \text { Humedad } \\
\text { relativa }\end{array}$ \\
\hline & & Vaisala. WXT520 AAB0BC10B0 & $\begin{array}{c} \pm 3 \% \mathrm{HR} \\
(0-90 \% \mathrm{HR}) \\
\pm 5 \% \mathrm{HR} \\
(90-100 \% \mathrm{HR})\end{array}$ & $\begin{array}{c}0-100 \% \\
\text { Humedad relativa }\end{array}$ \\
\hline & & Met One. Modelo 083E & $\begin{array}{c} \pm 2.0 \% \\
(0-100 \% \mathrm{HR})\end{array}$ & $\begin{array}{l}0-100 \% \text { Humedad } \\
\text { relativa }\end{array}$ \\
\hline \multirow{3}{*}{ Intensidad del viento } & \multirow{2}{*}{ Anemómetro (copas) } & Climatronics. Modelo F460 (P/N 100075) & $\pm 0.15- \pm 1.0 \mathrm{~m} / \mathrm{s}$ & $0-65 \mathrm{~m} / \mathrm{s}$ \\
\hline & & Met One. Modelo 010C & $0.07 \mathrm{~m} / \mathrm{s}$ & $0-22 \mathrm{~m} / \mathrm{s}$ \\
\hline & Sensor ultrasónico & Vaisala. WXT520 AAB0BC10B0 & $\begin{array}{c} \pm 0.3 \% \\
(10 \mathrm{~m} / \mathrm{s})\end{array}$ & $0-60 \mathrm{~m} / \mathrm{s}$ \\
\hline \multirow{3}{*}{ Dirección del viento } & \multirow[b]{2}{*}{ Veleta plana } & Climatronics. Modelo F460 (P/N 100076) & $\pm 2.0^{\circ}$ & $0^{\circ}-360^{\circ}$ \\
\hline & & Met One. Modelo 020C & $\pm 3.0^{\circ}$ & $\begin{array}{c}\left.0^{\circ}-257^{\circ} \text { (eléctrico }\right) \\
\left.0^{\circ}-360^{\circ} \text { (mecánico }\right)\end{array}$ \\
\hline & Sensor ultrasónico & Vaisala. WXT520 AAB0BC10B0 & $\pm 3.0^{\circ}$ & $0^{\circ}-360^{\circ}$ \\
\hline Radiación solar total & Radiómetro (con filtro) & Li-Cor & & \\
\hline Presión atmosférica & Sensor capacitivo & Vaisala. Modelo PTB101B & $\pm 0.5 \mathrm{mb}\left(20^{\circ} \mathrm{C}\right)$ & $600-1060 \mathrm{mb}$ \\
\hline
\end{tabular}

concentraciones de contaminantes en superficie. Sin embargo, por cuestiones de seguridad, el lanzamiento de las 18:00 h fue suspendido en varios días, ya que la velocidad del viento cerca de la superficie excedió los $10 \mathrm{~m} / \mathrm{s}$, lo cual representa un riesgo tanto para la instrumentación anclada al globo, como de ruptura del cable de sujeción del globo.

\section{Trayectorias de retroceso y avance y condiciones sinópticas}

Con el fin de aproximar las rutas que siguieron las masas de aire a diferentes niveles durante eventos de $\mathrm{O}_{3}$, se construyeron trayectorias de retroceso o retrotrayectorias (backward trajectories) y de avance (forward trajectories) utilizando el modelo Hybrid 
CUADRO II. CALENDARIO DE LANZAMIENTOS DE OZONOSONDEOS EN CHIP DURANTE LA CAMPAÑA DE MUESTREO

\begin{tabular}{|c|c|c|c|c|c|}
\hline \multicolumn{6}{|c|}{ OZONOSONDEOS EN CHIP } \\
\hline Fecha & $\begin{array}{c}\text { Hora de } \\
\text { lazamiento }\end{array}$ & $\begin{array}{l}\text { Observaciones } \\
\text { (altura máxima) }\end{array}$ & Fecha & $\begin{array}{c}\text { Hora de } \\
\text { lazamiento }\end{array}$ & Observaciones \\
\hline 27-mar-2018 & $\begin{array}{l}08: 00 \mathrm{~h} \\
12: 00 \mathrm{~h}\end{array}$ & $\begin{array}{l}950 \mathrm{~m} \\
900 \mathrm{~m}\end{array}$ & 07-abr-2018 & $\begin{array}{l}09: 00 \mathrm{~h} \\
13: 00 \mathrm{~h} \\
18: 00 \mathrm{~h}\end{array}$ & $\begin{array}{c}100 \mathrm{~m} \\
100 \mathrm{~m} \\
25 \mathrm{~m}\end{array}$ \\
\hline 28-mar-2018 & $\begin{array}{l}08: 00 \mathrm{~h} \\
12: 00 \mathrm{~h}\end{array}$ & $\begin{array}{l}960 \mathrm{~m} \\
100 \mathrm{~m}\end{array}$ & 09-abr-2018 & $\begin{array}{l}\text { 09:00 h } \\
13: 00 \mathrm{~h}\end{array}$ & $\begin{array}{l}60 \mathrm{~m} \\
80 \mathrm{~m}\end{array}$ \\
\hline 29-mar-2018 & 08:00 h & $75 \mathrm{~m}$ & $10-a b r-2018$ & $\begin{array}{l}\text { 09:00 h } \\
13: 00 \mathrm{~h} \\
18: 00 \mathrm{~h}\end{array}$ & $\begin{array}{c}100 \mathrm{~m} \\
50 \mathrm{~m} \\
--\end{array}$ \\
\hline 30-mar-2018 & $\begin{array}{l}08: 00 \mathrm{~h} \\
12: 00 \mathrm{~h} \\
18: 00 \mathrm{~h}\end{array}$ & $\begin{array}{l}75 \mathrm{~m} \\
75 \mathrm{~m} \\
80 \mathrm{~m}\end{array}$ & $11-a b r-2018$ & $\begin{array}{l}09: 00 \mathrm{~h} \\
13: 00 \mathrm{~h}\end{array}$ & $\begin{array}{l}100 \mathrm{~m} \\
98 \mathrm{~m}\end{array}$ \\
\hline 31-mar-2018 & $\begin{array}{l}08: 00 \mathrm{~h} \\
12: 00 \mathrm{~h}\end{array}$ & $\begin{array}{l}40 \mathrm{~m} \\
80 \mathrm{~m}\end{array}$ & $12-a b r-2018$ & $\begin{array}{l}\text { 09:00 h } \\
13: 00 \mathrm{~h} \\
18: 00 \mathrm{~h}\end{array}$ & $\begin{array}{l}25 \mathrm{~m} \\
75 \mathrm{~m} \\
50 \mathrm{~m}\end{array}$ \\
\hline $01-a b r-2018$ & 08:00 h & $75 \mathrm{~m}$ & 13-abr-2018 & $\begin{array}{l}\text { 09:00 h } \\
13: 00 \mathrm{~h}\end{array}$ & $\begin{array}{l}50 \mathrm{~m} \\
80 \mathrm{~m}\end{array}$ \\
\hline 02-abr-2018 & 09:00 h & $80 \mathrm{~m}$ & $16-a b r-2018$ & $\begin{array}{l}09: 00 \mathrm{~h} \\
13: 00 \mathrm{~h} \\
18: 00 \mathrm{~h}\end{array}$ & $\begin{array}{c}60 \mathrm{~m} \\
100 \mathrm{~m} \\
75 \mathrm{~m}\end{array}$ \\
\hline 03-abr-2018 & 09:00 h & $75 \mathrm{~m}$ & $17-a b r-2018$ & 09:00 h & $100 \mathrm{~m}$ \\
\hline 04-abr-2018 & $\begin{array}{l}09: 00 \mathrm{~h} \\
13: 00 \mathrm{~h}\end{array}$ & $80 \mathrm{~m}$ & 18 -abr-2018 & $\begin{array}{l}09: 00 \mathrm{~h} \\
13: 00 \mathrm{~h} \\
18: 00 \mathrm{~h}\end{array}$ & $\begin{array}{l}80 \mathrm{~m} \\
50 \mathrm{~m} \\
50 \mathrm{~m}\end{array}$ \\
\hline 05-abr-2018 & $\begin{array}{l}\text { 09:00 h } \\
13: 00 \mathrm{~h} \\
18: 00 \mathrm{~h}\end{array}$ & $\begin{array}{c}60 \mathrm{~m} \\
60 \mathrm{~m} \\
--\end{array}$ & 19-abr-2018 & $\begin{array}{l}09: 00 \mathrm{~h} \\
13: 00 \mathrm{~h}\end{array}$ & $\begin{array}{c}75 \mathrm{~m} \\
100 \mathrm{~m}\end{array}$ \\
\hline 06-abr-2018 & $\begin{array}{l}09: 00 \mathrm{~h} \\
12: 00 \mathrm{~h}\end{array}$ & $\begin{array}{l}97 \mathrm{~m} \\
40 \mathrm{~m}\end{array}$ & & & \\
\hline
\end{tabular}

Single Particle Lagrangian Integrated Trajectory (HYSPLIT) de la National Oceanic and Atmospheric Administration (NOAA) (Stein et al. 2015, Rolph et al. 2017). El modelo reproduce la posición (longitud y latitud) y elevación de la parcela antes de llegar (retroceso) o después de partir (avance) de posiciones geográficas de interés, en este caso de los sitios HUAQ, CHIP y AMOZ. La exactitud y limitaciones de los cálculos de las trayectorias están determinadas por la resolución espacial y temporal de las observaciones meteorológicas, por el modelo de campos de vientos utilizado para la interpolación y por las suposiciones de simplificación del modelo de trayectoria. Los datos de los campos meteorológicos para correr el modelo fueron seleccionados de archivos de datos "Reanalysis" generados por el Global National Weather Service's National Centers for Environmental Prediction (NCEP) para las fechas de los eventos. Las trayectorias se obtuvieron en forma interactiva con la versión en línea del HYSPLIT en el sitio del Air Resources Laboratory de la NOAA. Se seleccionaron $12 \mathrm{~h}$ de retroceso y $12 \mathrm{~h}$ de avance para dos niveles de altura de paso de estas trayectorias sobre los sitios en la superficie de $100 \mathrm{~m}$ y $500 \mathrm{~m}$. Asimismo, se utilizó el modelo vertical de velocidad en modo "Archived Surface Analysis" generado por el Weather Prediction Center y disponible al público a través de la NOAA para identificar el tipo de condición sinóptica sobre el centro de México en eventos seleccionados. 


\section{RESULTADOS}

\section{Meteorología sinóptica}

Durante la campaña de mediciones se presentaron variaciones en las condiciones sinópticas alternando entre sistemas de baja y alta presión sobre la parte central del país. La figura 3 muestra ejemplos de los sistemas sinópticos típicos sobre Norteamérica para los días 17,19 y 21 con sistemas de tipo transición el primer día y de alta presión los otros.

\section{Meteorología en superficie}

La figura 4 muestra las rosas de viento en superficie para los sitios de CHIP, HUAQ y AMOZ en periodos de $6 \mathrm{~h}$ para toda la campaña. Los vientos de superficie observados en cada sitio reflejaron el efecto de la combinación de vientos regionales de tipo valle-montaña y su interacción con los vientos de altura, tal y como previamente lo describen Lauer y Klaus (1975). Durante la madrugada y hasta antes del amanecer, se observaron flujos predominantes provenientes del norte y noreste en todos los casos, influenciados por vientos de escurrimiento de la Malinche y de la Sierra Nevada. Durante la mañana y antes del mediodía, ocurre un cambio en la dirección de los vientos como resultado de las diferencias térmicas en el valle y las laderas en torno al VPT en HUAQ y AMOZ. En HUAQ no se observó este patrón de cambio, manteniéndose flujos del NE en la noche, ocurriendo el escurrimiento de masas de aire hacia la parte más baja y caliente de la región.

No obstante, del mediodía al atardecer, ocurre un cambio completo en la dirección del viento en superficie, ahora hacia el NE en los tres sitios. Este cambio en la dirección parece tener el mismo origen que el patrón observado en la parte occidental de la Sierra Nevada, en donde de forma similar se presentan flujos de retorno vespertinos del valle Cuernavaca-Cuautla (VCC) al valle de México por la influencia de un gradiente de presión inducido térmicamente entre el VPT y el VCC que domina el equilibrio de momentum en la advección de las masas de aire entre Cuautla e Izúcar de Matamoros (Fast y Zhong 1998). Al anochecer y hasta la media noche los vientos del sur-suroeste (S-SO) se debilitan hasta restablecer el flujo de vientos catabáticos del norte-noreste (N-NE).

\section{Mediciones en la vertical}

La figura 5 muestra ejemplos de perfiles verticales de $\mathrm{O}_{3}$, temperatura, humedad relativa, dirección y velocidad de viento observados con el globo cautivo
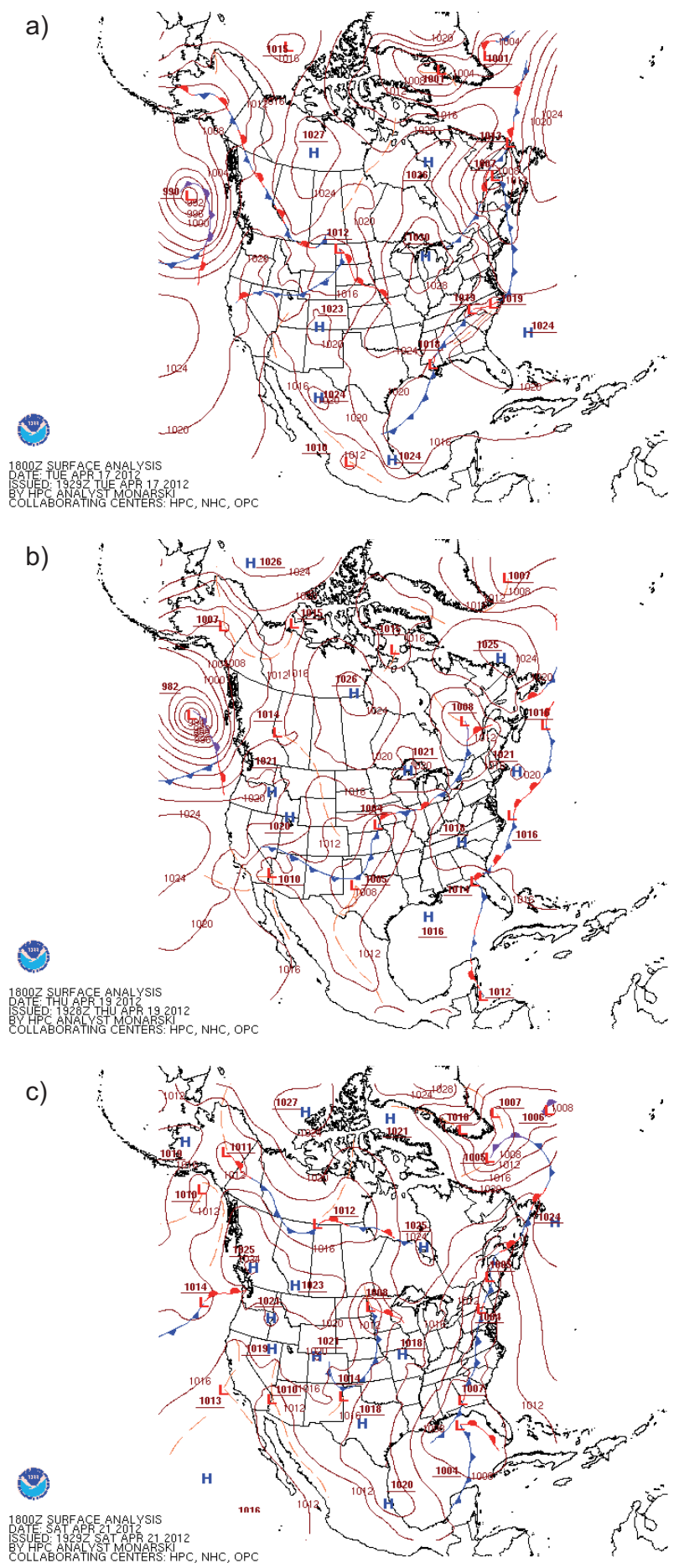

Fig. 3. Condiciones sinópticas en superficie a las 12:00 h (hora local) para, a) 17 ; b) 19 y c) 21 de abril. Análisis de superficie tomado de National Oceanic and Atmospheric Administration (NOAA 2014) 

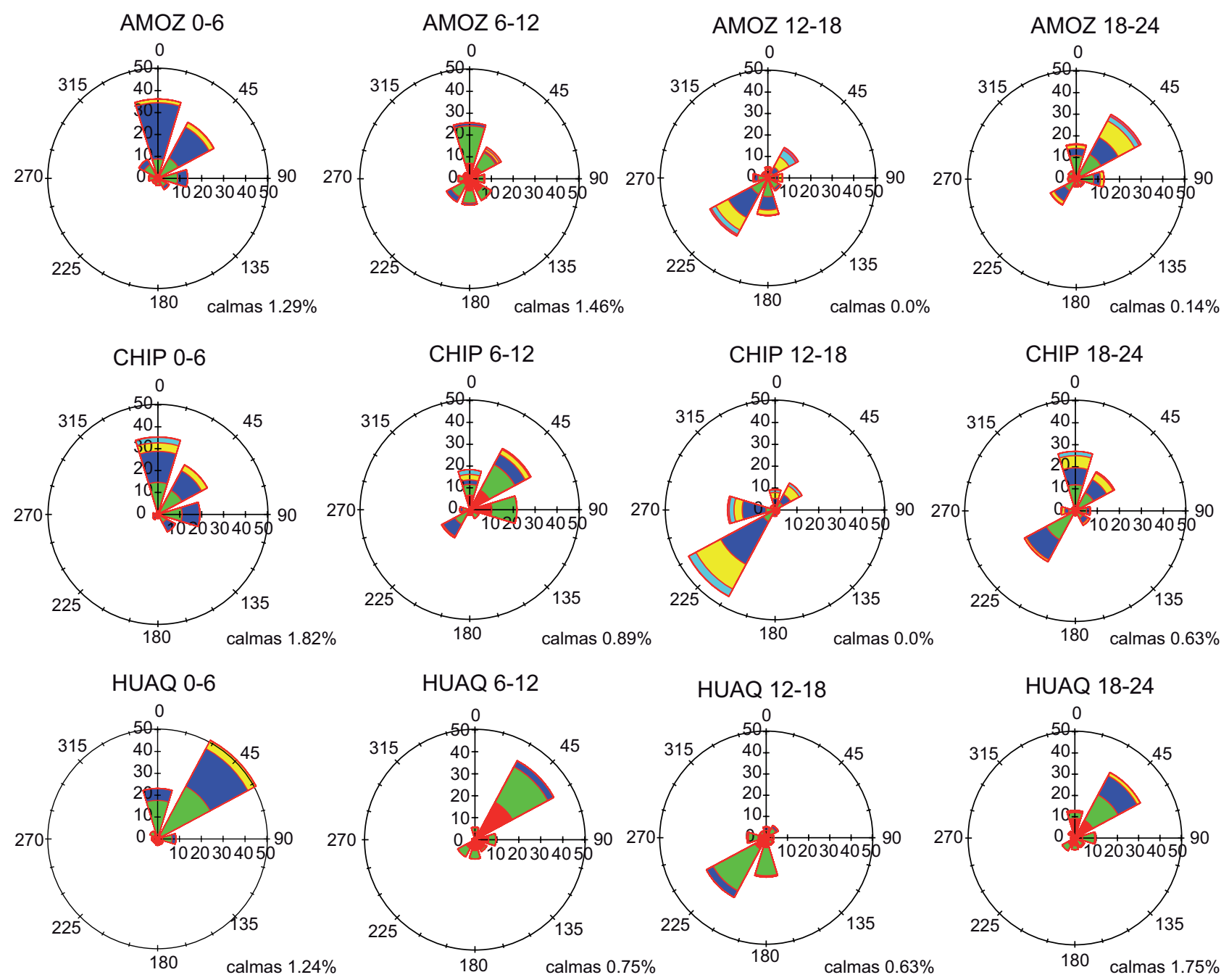

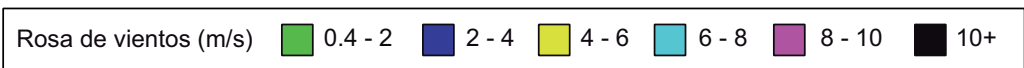

Fig. 4. Rosas de vientos para Amozoc (AMOZ), Chipilo (CHIP) y Huaquechula (HUAQ) construidas con una resolución temporal de 5 min del 30 de marzo al 27 de abril de 2012

instrumentado para un día típico con influencia de un sistema sinóptico de transición (17 de abril) y otro de alta presión (19 de abril).

Bajo influencia del sistema sinóptico de transición hacia alta presión, el perfil de viento muestra una clara dominancia del NE y del E en prácticamente todo el perfil vertical medido con flujos provenientes del E-NE (Golfo de México). El perfil de temperatura muestra un patrón muy estable con dos capas de inversión, una por arriba de $\operatorname{los} 300 \mathrm{~m}$ y otra a $850 \mathrm{~m}$ sobre la superficie. El perfil de $\mathrm{O}_{3}$ presenta un incremento inicial de $\sim 0.040 \mathrm{ppm}$ a $\sim 0.050 \mathrm{ppm}$ para mantenerse relativamente constante, hasta que al llegar a los $300 \mathrm{~m}$ aumenta súbitamente hasta las $0.060 \mathrm{ppm}$ y se mantiene en ese régimen hasta por arriba de los $1000 \mathrm{~m}$ sobre la superficie.

Por otro lado, bajo la influencia de alta presión el viento en la superficie proviene del NE para cambiar rápidamente a vientos del S-SO en torno a los $300 \mathrm{~m}$ y paulatinamente a vientos del $\mathrm{O}$ y $\mathrm{NO}$ conforme se incrementa la altura. El perfil de temperatura muestra condiciones parcialmente inestables con la presencia de una capa estable entre los 300 y $400 \mathrm{~m}$ aproximadamente.

$\mathrm{El}$ perfil de $\mathrm{O}_{3}$ se mantuvo estable en torno a las $0.020 \mathrm{ppm}$ en los primeros $200 \mathrm{~m}$ para posteriormente 

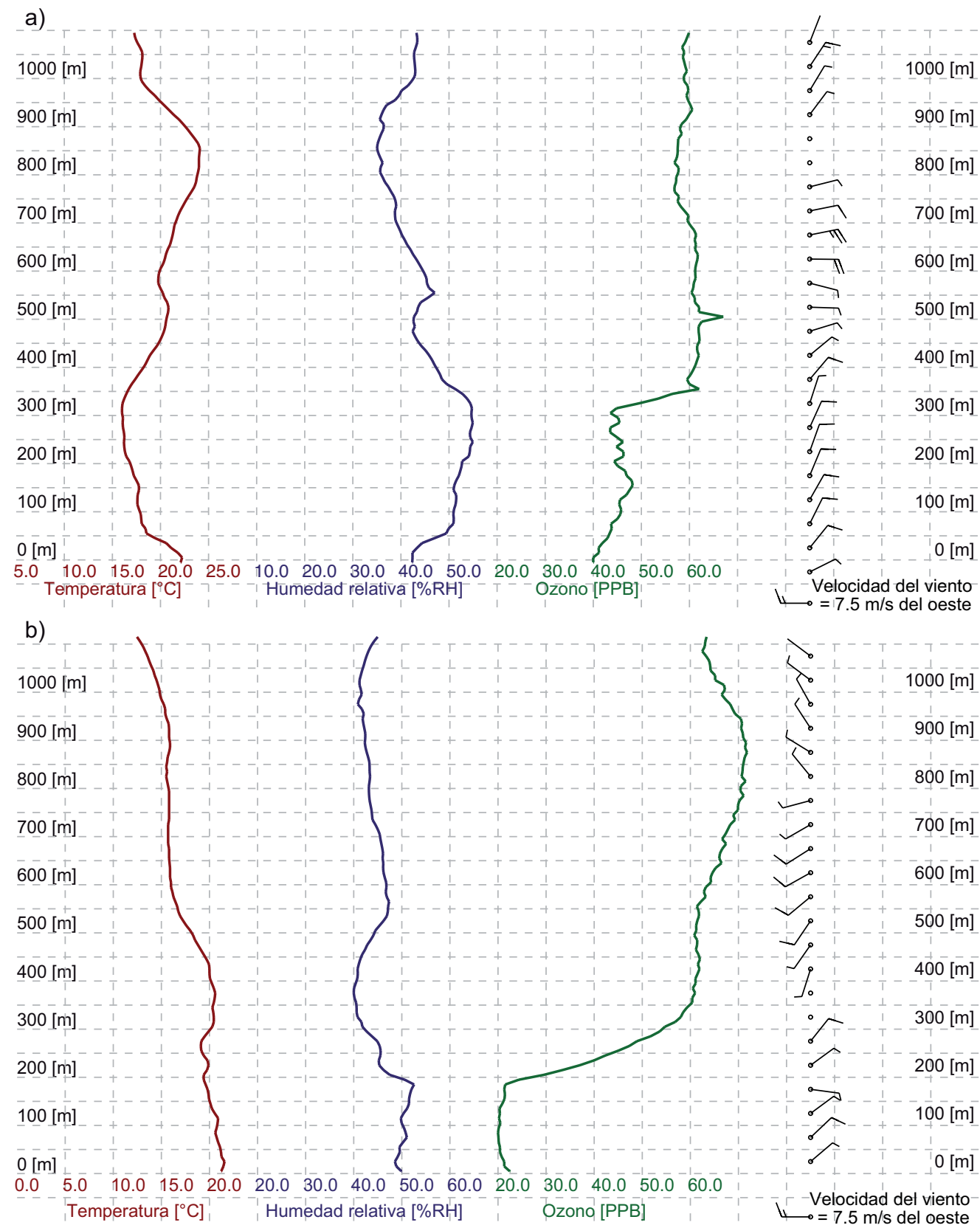

Fig. 5. Perfiles verticales de temperatura, humedad relativa, ozono y dirección e intensidad del viento del: (a) 17 y (b) 19 de abril de 2012, ambos para lanzamientos del globo cautivo a las 09:00 h en CHIP

incrementarse rápidamente hasta alcanzar las 0.060 ppm antes de los $400 \mathrm{~m}$ en coincidencia con el cambio en la dirección del viento asociado con el flujo proveniente del suroeste (SO) de México (Océano Pacífico), llegando a alcanzar hasta poco más 0.080 ppm en torno a los $800 \mathrm{~m}$ sobre la superficie.

Los perfiles analizados indican que cuando el viento sopla del Golfo de México (E-NE), el $\mathrm{O}_{3}$ en la altura es menor que cuando el viento proviene del Pacífico (SO), lo que sugiere que hay un potencial de contribución importante de $\mathrm{O}_{3}$ en esta región del Altiplano Mexicano, posiblemente de la tropósfera libre, que puede ser incorporado a la superficie al desarrollarse la capa de mezcla, en forma similar a lo reportado por Thompson et al. (2008) durante la campaña MILAGRO. 


\section{Mediciones de $\mathrm{O}_{3}$ en superficie}

Las concentraciones promedio horario de $\mathrm{O}_{3}$ en superficie en AMOZ, CHIP y HUAQ del 30 de marzo al 27 de abril de 2012 se presentan en la figura 6. Los periodos sin datos en esta figura se deben a problemas de suministro de energía eléctrica.

En general, se observó una gran correspondencia entre las mediciones de $\mathrm{O}_{3}$ en todos los sitios con pequeñas diferencias en su magnitud (Fig. 6), lo que implica que los tres sitios pudieron haber registrado el paso de las mismas masas de aire, y que las variaciones diurnas estuvieron influenciadas por las condiciones meteorológicas de la región y por el mismo sistema sinóptico.

Se observaron dos episodios que sumaron un total de $5 \mathrm{~h}$ de excedencia a las $0.095 \mathrm{ppm}$ en CHIP (SSA, 2014), y un episodio de $1 \mathrm{~h}$ en AMOZ. Asimismo, se encontró que conforme transcurrió el mes de abril, los niveles de $\mathrm{O}_{3}$ fueron cada vez mayores de acuerdo con lo esperado durante la temporada de $\mathrm{O}_{3}$ en el centro de México, con días con mayor temperatura e insolación (Molina et al. 2010). Las rosas de contaminación (Fig. 7) para concentraciones promedio de 5 min en los tres sitios durante la campaña muestran que las mayores concentraciones de $\mathrm{O}_{3}$ ocurrieron en el periodo comprendido entre las 12:00 y las 18:00 $\mathrm{h}$ cuando el viento provino del S-SO con niveles incluso por arriba de las $0.080 \mathrm{ppm}$. En el periodo de 18:00 a 24:00 h todavía se registraron niveles mayores a $0.060 \mathrm{ppm}$.

En AMOZ y HUAQ estos niveles están asociados a vientos de retorno del NE, mientras que en CHIP hay una persistencia de vientos después del atardecer del SO y de retorno provenientes del sector N-NE una vez entrada la noche. En general, las parcelas de aire todavía contenían $\mathrm{O}_{3}$ remanente que previamente había pasado a través del valle hacia el N-NE. En el último caso, las parcelas de aire de retorno en CHIP contienen una menor cantidad de $\mathrm{O}_{3}$ debido a que durante su paso por la ciudad de Puebla ocurre una remoción mayor de este contaminante por titulación con emisiones frescas urbanas de NO. En el periodo de las 00:00 a 06:00 h dominaron en general concentraciones de $\mathrm{O}_{3}$ menores a las medidas antes de las 24:00 h pero ligeramente mayores a las 0.040 ppm. Finalmente, entre las 06:00 h y hasta las 12:00 $\mathrm{h}$ persiste la influencia de vientos del norte y noreste acarreando parcelas de aire con niveles relativamente bajos de $\mathrm{O}_{3}$, pero con evidencia de contribuciones tanto locales como regionales, en particular CHIP y HUAQ.

\section{Trayectorias de parcelas de aire}

En la figura 8 se muestra el resultado de simulaciones de trayectorias de retroceso (TR) y avance (TA) de parcelas de aire realizadas con HYSPLIT para los niveles de arribo a los sitios de estudio de $100 \mathrm{~m}$ y $500 \mathrm{~m}$ a las $13 \mathrm{~h}$ (hora local). La influencia del sistema sinóptico de transición hacia alta presión se observa en la dinámica de los flujos de viento de ese día. Las trayectorias de retroceso (TR) de las parcelas de aire que llegan a la zona de estudio a los dos niveles provienen del NE del VPT, y cuatro de ellas se ubicaban $12 \mathrm{~h}$ antes por arriba de los 500 $m$ sobre la superficie según se muestra en la parte inferior de las tres trayectorias del lado izquierdo. Sin embargo, mientras que las parcelas de aire que pasan a los $100 \mathrm{~m}$ siguen su trayectoria de avance (TA) hacia el SO elevándose para bordear la Sierra Nevada, las que pasaron sobre los sitios en el nivel de los $500 \mathrm{~m}$ giran en el sentido de las manecillas del reloj para retornar hacia el NE elevándose

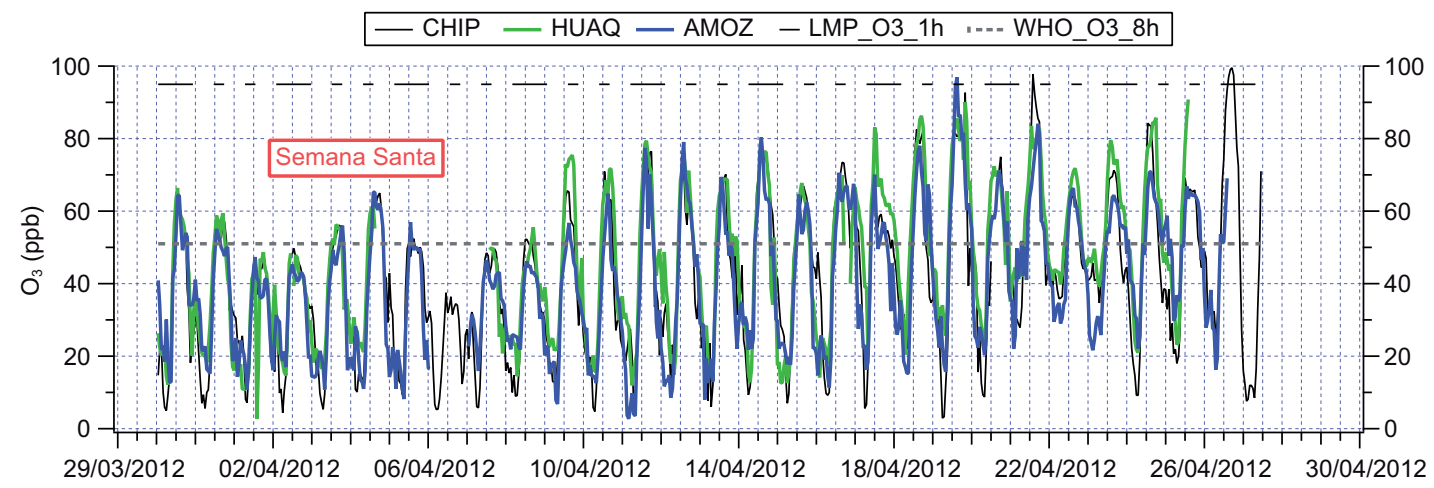

Fig. 6. Serie de tiempo para $\mathrm{O}_{3}$ en AMOZ, CHIP y HUAQ en superficie durante la campaña de mediciones del 30 de marzo al 27 de abril de 2012. El recuadro naranja discontinuo denota el periodo correspondiente a Semana Santa, y la línea negra discontinua representa el límite máximo permisible de concentración promedio horario (0.095 ppm) en México (NOM-020- SSA1-2014) 

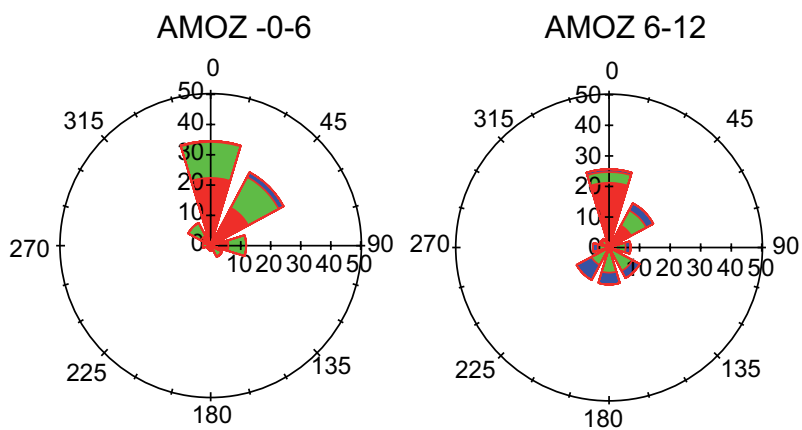

CHIP $-0-6$

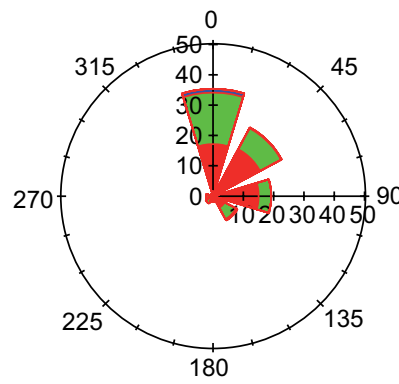

HUAQ $-0-6$

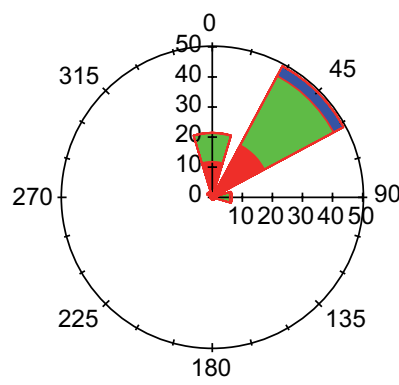

CHIP 6-12

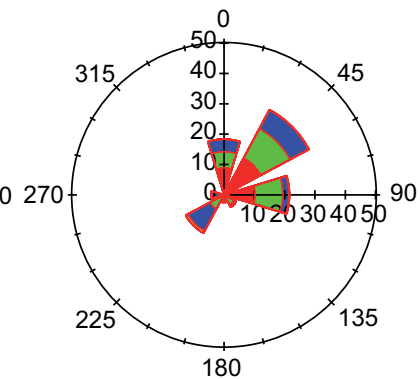

HUAQ 6-12

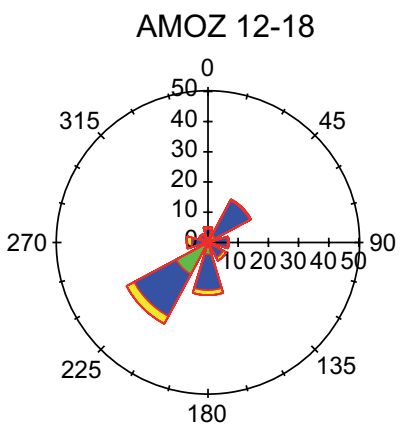

CHIP 12-18

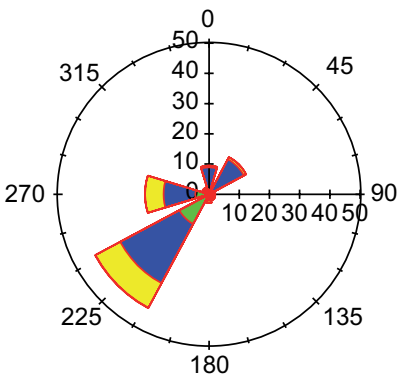

HUAQ 12-18

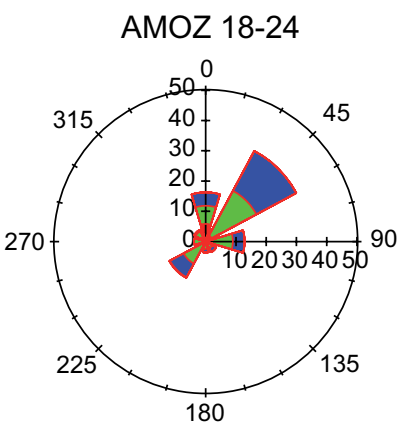

CHIP 18-24

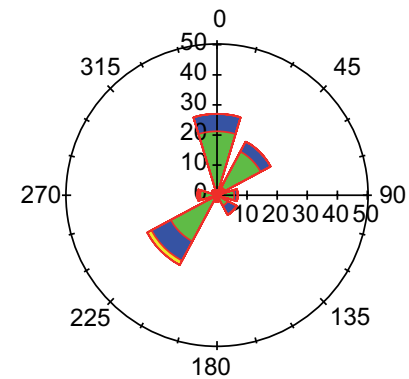

HUAQ 18-24
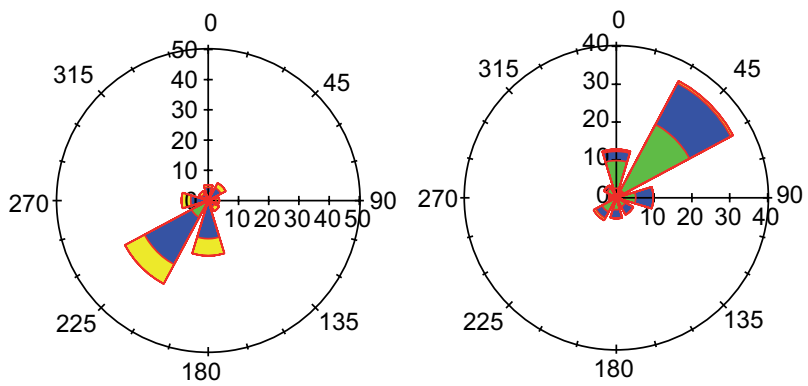

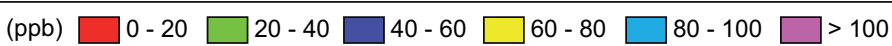

Fig. 7. Rosas de $\mathrm{O}_{3}$ para $\mathrm{AMOZ}$, CHIP y HUAQ, divididas en tres grupos con intervalos de 00:00-06:00 h, 06:00-18:00 h y 18:0024:00 h para toda la campaña de medición

para cruzar la Sierra Madre Oriental por arriba de los $5000 \mathrm{~m}$ (trayectorias del lado derecho Fig. 8). El aporte de $\mathrm{O}_{3}$ vespertino a los sitios de estudio HUAQ y CHIP fue posiblemente de la misma zona urbana de Puebla, mientras que para AMOZ, que aparentemente no recibió recirculación del VPT, de la conurbación de la ciudad de Tlaxcala al N-NE de la Malinche. Asimismo, los tres sitios pudieron recibir contribuciones de $\mathrm{O}_{3}$ de la tropósfera libre mediante acarreo de las parcelas de aire que inicialmente estaban por arriba de los 1000 m (3100 msnm), hacia capas más bajas, y que unas horas antes del mediodía se ubicaban por abajo de los 400 $m$ antes de pasar los sitios de estudio, tal y como se observa en el perfil vertical de las TR.
El día 19 de abril y ya bajo la influencia del sistema sinóptico de alta presión, las TR muestran claramente una recirculación lenta de masas de aire ubicadas al sur del VPT hacia la zona urbana de Puebla, a niveles que inician incluso por debajo de los $500 \mathrm{~m}$ de altura. Los niveles de $\mathrm{O}_{3}$ que se observaron en los tres sitios a partir de mediodía pudieron ser concentraciones recirculadas dentro de masas de aire envejecidas del día anterior que permanecieron dentro del VPT. El sitio AMOZ recibe el mayor impacto de esta recirculación de acuerdo con la TR correspondiente por su cercanía con la ciudad. Los niveles de $\mathrm{O}_{3}$ observados en los tres sitios al atardecer son contribuciones tanto de este $\mathrm{O}_{3}$ recirculado con el provenientes del VCC. Horas más tarde, estas 


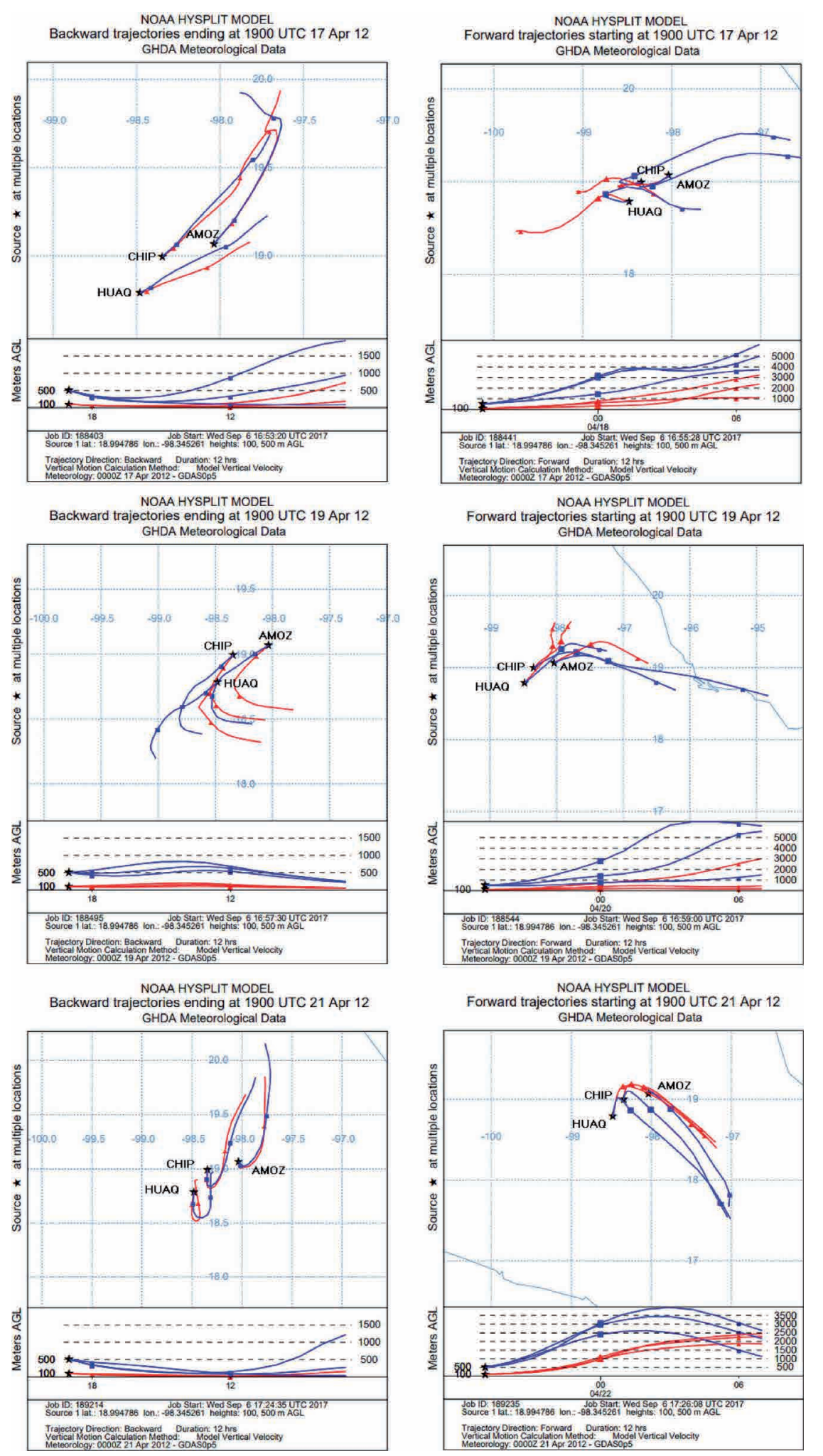

Fig. 8. Trayectorias de retroceso (panel izquierdo), y de avance (panel derecho), para los días 17, 19 y 21 de abril de 2012 a las 13:00 h (hora local), con niveles de alturas de arribo o inicio de las parcelas de aire sobre el nivel del suelo a los $100 \mathrm{~m}$ (color rojo) y $500 \mathrm{~m}$ (color azul), usando el modelo HYSPLIT de la National Oceanic and Atmospheric Administration (NOAA) 
masas de aire continuaron con ese giro en dirección al NE-E-SE en ascenso sobre la Sierra Madre Oriental.

El día 21 continúa la influencia del sistema sinóptico de alta presión que se distingue con la recirculación de masas de aire mostrada en las TR, aunque ya debilitado. Las parcelas de aire provienen del N-NE con respecto al VPT con trayectorias que inician en general por abajo de los $500 \mathrm{~m}$ de altura sobre la superficie media, por lo que pudieron acarrear $\mathrm{O}_{3}$ y precursores provenientes de la zona conurbada de Tlaxcala, siendo su mayor su influencia en AMOZ, mientras que en HUAQ y CHIP la mayor contribución pudo provenir de la recirculación de $\mathrm{O}_{3}$ fresco de la propia zona urbana de Puebla. La recirculación es prácticamente sobre el centro del VPT tal y como lo muestran las TA, estableciendo un flujo del $\mathrm{SO}$ al NNE que pudo estar enriquecido con contribuciones del VCC. Entrada la noche las parcelas se ubican ya en ascenso hacia el SE sobre la Sierra Madre Oriental.

A diferencia de las trayectorias de la figura 8 cuya hora de llegada y partida a los sitios de estudio es a las 13:00 h (hora local), las trayectorias de la figura 9 presentan las TR estimadas con llegada a las 20:00 h (hora local) a los sitios de estudio para los tres sitios durante la ocurrencia de eventos nocturnos. Las parcelas de aire que pasaron sobre la zona de estudio en ambos niveles (100 y $500 \mathrm{~m}$ ) a las 20:00 h el $17 \mathrm{de}$ abril aún provienen del NE del VPT, igual que las parcelas que previamente se analizaron para las 13:00 h. Dos de las parcelas con arribo en los $500 \mathrm{~m}$ en el análisis de trayectorias de las 20:00 h, se ubicaban a más de $2000 \mathrm{~m}$ de altura ( $4100 \mathrm{msnm}) 12 \mathrm{~h}$ antes, mientras que $4 \mathrm{~h}$ antes de pasar por los sitios, las parcelas ya se ubicaban dentro del nivel de los $500 \mathrm{~m}$. Las masas de aire en el nivel de los $100 \mathrm{~m}$ prácticamente se mantuvieron por debajo de este nivel. Por ello es factible que el $\mathrm{O}_{3}$, tanto de la troposfera libre como de las masas de aire, se mantuviera recirculando en el VPT durante la tarde del 17 de abril, y de esta manera haya contribuido a mantener niveles relativamente altos de $\mathrm{O}_{3}$ al anochecer. Para el 19 de abril, con condiciones de alta presión, las trayectorias de masas de aire que llegan en la noche a los tres sitios sugieren claramente la contribución de $\mathrm{O}_{3}$ de la troposfera libre y del VCC en los dos niveles de arribo. En este caso, las que pasan al nivel de $500 \mathrm{~m}$, provenían $6 \mathrm{~h}$ antes de por arriba de los $1000 \mathrm{~m}$ de altura $(\sim 3100$ $\mathrm{msnm}$ ), mientras que las del nivel de $100 \mathrm{~m}$ por arriba de aproximadamente $300 \mathrm{~m}(\sim 2400 \mathrm{msnm})$. Estas últimas pudieron acarrear de regreso el $\mathrm{O}_{3}$ que horas antes había sido transportado hacia el SO del VPT. En cuanto al evento nocturno del 21 de abril, se observa una posible confluencia de masas de aire del NE-N para AMOZ y del SE en CHIP y HUAQ. Las TR de las masas de aire que arribaron en los niveles de 100 m y $500 \mathrm{~m}$ en CHIP y HUAQ se mantuvieron bajo transporte advectivo cerca de la superficie recolectando $\mathrm{O}_{3}$ que había sido recirculado durante el día dentro del VPT y que al atardecer se expandieron dentro de la capa de mezcla poco antes de pasar por estos sitios.

\section{DISCUSIÓN}

Los resultados de las mediciones realizadas muestran que el valle de Puebla está siendo en general receptor de parcelas ricas en $\mathrm{O}_{3}$ ajenas a la ZMVPT, en combinación con la recirculación de niveles de este contaminante que es retornado al VPT. Asimismo, se observa que los vientos en la altura provenientes del Pacífico influyen con mayor intensidad sobre los niveles de $\mathrm{O}_{3}$ en superficie que los provenientes del norte y Golfo de México. La figura 10 muestra la serie de tiempo y la relación entre las concentraciones de $\mathrm{O}_{3}$, $\mathrm{NO}_{2}$ y dirección del viento de las concentraciones de $\mathrm{O}_{3}$ observadas los días 17, 19 y 21 de abril. Se observa que hay similitud en los perfiles de $\mathrm{O}_{3}$ en los tres sitios desde aproximadamente las 07:00 h con un nivel de $\mathrm{O}_{3}$ más bajo para el día 19 de abril y más alto para el 21 de abril. Asimismo, se observan algunas diferencias en la rapidez del incremento en este contaminante, ocurriendo el máximo más temprano durante la condición sinóptica de transición (17 de abril), que durante la influencia de alta presión (19 de abril y 21 de abril). Se hace una discusión más detallada de cada uno de los días en los siguientes párrafos.

\section{7 de abril}

Este día el viento dominante tanto en superficie como en la altura fue del NE-N y los niveles diurnos de $\mathrm{O}_{3}$ fueron relativamente bajos, indicando un transporte de masas de aire del norte del Golfo de México hacia el VPT. De acuerdo con la figura 10, durante la madrugada el viento provino del $\mathrm{N}$ en AMOZ; del sector N-NE-E en CHIP; y del NE en $\mathrm{HUAQ}$, con niveles de $\mathrm{O}_{3}$ remanentes cercanos a la concentración de fondo del orden de $\sim 0.040 \mathrm{ppm}$ en AMOZ, de cerca de $0.050 \mathrm{ppm}$ para CHIP, y por arriba de 0.060 ppm en HUAQ. Por la mañana, la mayor concentración de $\mathrm{NO}_{2}$ se observa en CHIP por la influencia de la Ciudad de Puebla, aunque sus niveles apenas alcanzan $0.040 \mathrm{ppm}$. Por comparación con la CDMX, en esta zona urbana se registran niveles del orden de 0.100 a 0.120 ppm de $\mathrm{NO}_{2}$ durante la mañana (SEDEMA2018). La producción y acumulación de $\mathrm{O}_{3}$ inicia poco después de las 07:00 h 


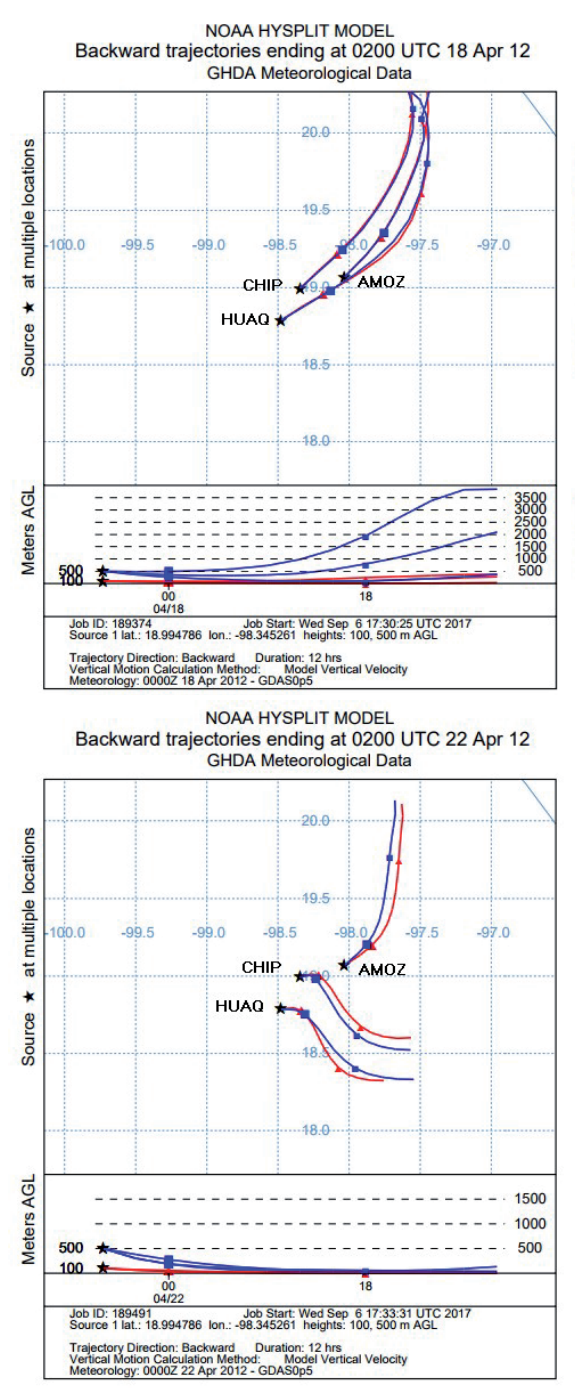

en los tres sitios. Sin embargo, la rapidez de su incremento observado entre las 08:00 h y cerca de las 11:00 h, no parecen estar acorde al $\mathrm{NO}_{2}$ disponible para alcanzar las concentraciones de $\mathrm{O}_{3}$ registradas, por lo que la posibilidad alterna más viable pudo ser la adición de $\mathrm{O}_{3}$ remanente en la altura por mecanismo de acarreo hacia la superficie cuando se rompe la capa de inversión. Este efecto se observa con el cambio de dirección del viento en AMOZ y la caída súbita de $\mathrm{NO}_{2}$ por efecto de dilución en los tres sitios. Este proceso de acarreo persiste hasta cerca del mediodía siendo la rapidez de acumulación de $\mathrm{O}_{3}$ muy similar en los tres sitios. El máximo de $\mathrm{O}_{3}$ se registró aproximadamente a las 11:00 h y con mayor concentración en HUAQ debido al efecto acumulativo de $\mathrm{O}_{3}$ en las parcelas de aire tanto del $\mathrm{O}_{3}$ recién formado como del mezclado de las capas

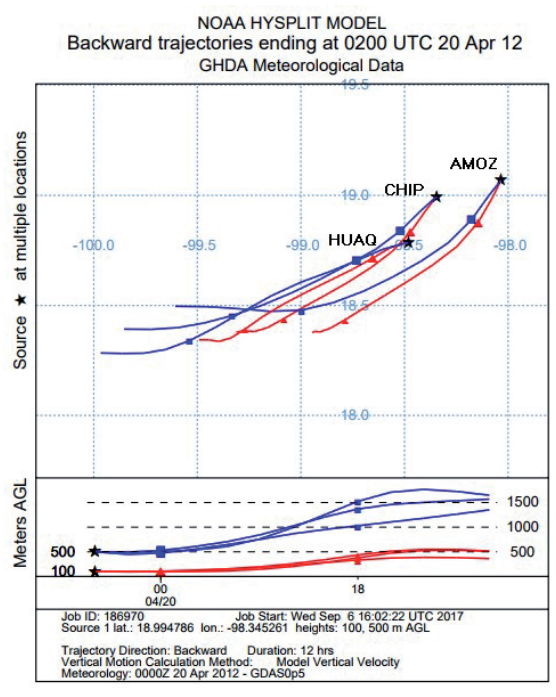

Fig. 9. Trayectorias de retroceso para los días 17,19 y 21 de abril de 2012, a las 20:00 h (hora local) con alturas de arribo sobre el nivel del suelo a los $100 \mathrm{~m}$ (color rojo) y $500 \mathrm{~m}$ (color azul), utilizando el modelo HYSPLIT de la National Oceanic and Atmospheric Administration (NOAA)

superiores conforme estas se desplazaron a lo largo de la trayectoria en dirección NE a SO.

Después de un pico de $\mathrm{O}_{3}$ registrado en los tres sitios cerca de las 12:00 h, los niveles de $\mathrm{O}_{3}$ disminuyen y permanecen prácticamente en la misma concentración en los tres sitios hasta antes de las 18:00 $\mathrm{h}$ cuando ocurre un nuevo cambio, situación previamente asociada a la recirculación de parcelas de aire en el VPT. Mientras que en AMOZ hay una clara influencia de remoción de $\mathrm{O}_{3}$ por abundancia de $\mathrm{NO}$, que se refleja en una mayor concentración de $\mathrm{NO}_{2}$. En CHIP y HUAQ las concentraciones de $\mathrm{O}_{3}$ se sostienen un mayor tiempo por arriba de las $0.050 \mathrm{ppm}$, al no existir emisiones de $\mathrm{NO}$ en abundancia. Las trayectorias de las 20:00 h mostradas en la figura 9 explican el posible origen de los niveles nocturnos de $\mathrm{O}_{3}$ mediante acarreo de capas superiores. 


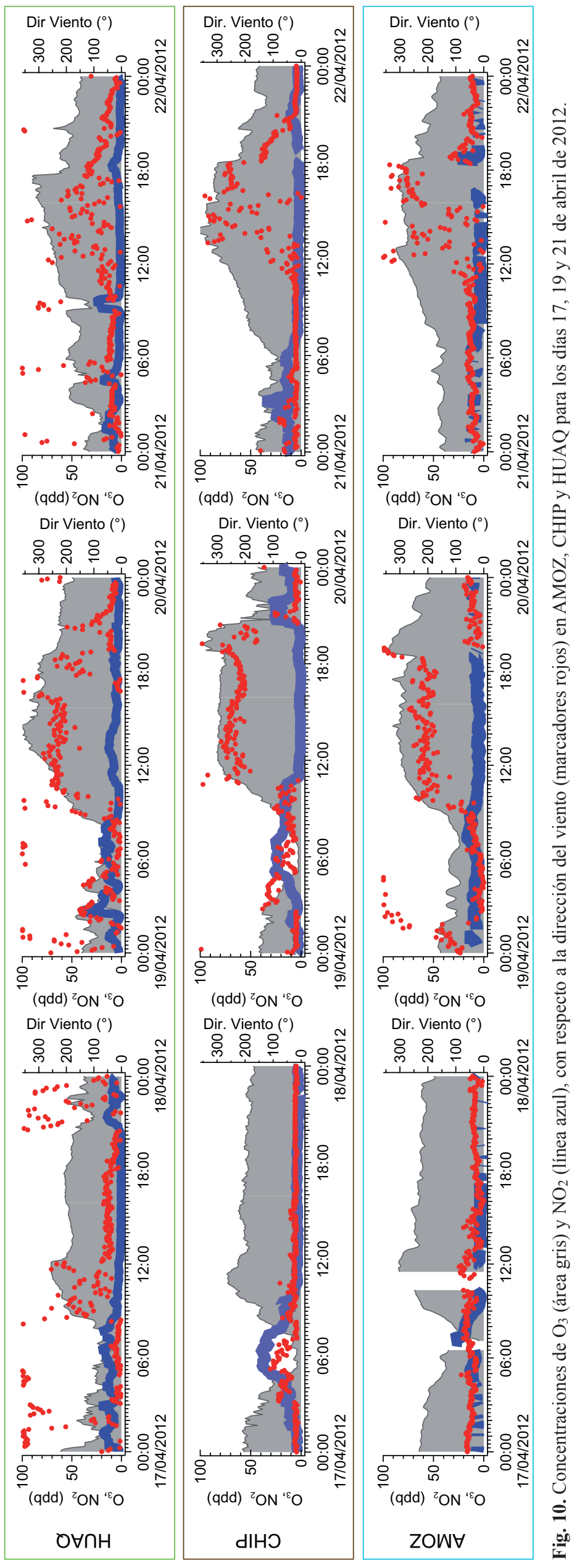




\section{9 de abril}

El viento dominante durante la madrugada fue del N-NE registrándose valores de $\mathrm{O}_{3}$ por abajo de la concentración de fondo, lo que sugiere que diversos mecanismos nocturnos de remoción como la titulación con NO fueron comunes en el valle, siendo más notable en torno a AMOZ. Al amanecer este fenómeno fue ligeramente más intenso en AMOZ y CHIP, aunque los niveles de $\mathrm{NO}_{2}$ apenas rebasaron las $0.020 \mathrm{ppm}$. El efecto del rompimiento de la capa de inversión y del acarreo a la superficie de $\mathrm{O}_{3}$ atrapado arriba de la capa de inversión se aprecia entre las 07:00 y las 08:00 h con la ocurrencia de un incremento súbito en $\mathrm{O}_{3}$. La rapidez de incremento de $\mathrm{O}_{3}$ fue abrupta y muy similar en los tres sitios y se sostuvo mientras la dirección de proveniencia del viento fue del NE, tal y como ocurrió el día 17 de abril. Sin embargo, cerca de las 11:00 h se identifica un cambio completo en la dirección del viento del $\mathrm{NE}$ al SSO-SO que es registrado en los tres sitios y que es observado en las trayectorias de retroceso de la figura 8. Las parcelas de aire que horas antes se dirigían al $\mathrm{SO}$ vienen ahora de regreso en el proceso de recirculación identificado acompañadas con un incremento adicional en los niveles de $\mathrm{O}_{3}$, posiblemente de ozono envejecido sumado al ozono fresco del nuevo día.

Este día se registra un aumento de $\mathrm{O}_{3}$ poco después de las 13:00 h en los tres sitios una vez establecido el flujo del $\mathrm{SO}$ con la adición de $\mathrm{O}_{3}$ regional al recirculado. En HUAQ el aumento para la hora antes señalada alcanza el orden de las $0.080 \mathrm{ppm}$; en tanto que en CHIP casi se alcanzan las $0.090 \mathrm{ppm}$, mientras que en $\mathrm{AMOZ}$ las parcelas enriquecidas con $\mathrm{O}_{3}$ de la Ciudad de Puebla hacen que los niveles de $\mathrm{O}_{3}$ lleguen por arriba de las $0.100 \mathrm{ppm}$. Después de este periodo ya no hay mayor acumulación de $\mathrm{O}_{3}$ por aproximadamente $5 \mathrm{~h}$ más y en los tres sitios continúan arribando parcelas de aire recirculadas del VPT conteniendo del orden de $0.070 \mathrm{ppm} \mathrm{de} \mathrm{O}_{3}$. No obstante, al anochecer ocurre un repentino cambio en la dirección del viento. Mientras que en AMOZ y HUAQ se establece un flujo del NE, en CHIP el viento proviene todavía del sector SSO-S-SSE. Si bien, en AMOZ este cambio en la dirección va acompañado con una reducción en el contenido de $\mathrm{O}_{3}$ en las parcelas de aire, debido a la posibilidad de que el viento provenga de una zona rural sin aportes de esta especie, en CHIP y HUAQ hay un incremento súbito inusual de $\mathrm{O}_{3}$ que alcanza poco más de $0.090 \mathrm{ppm}$ entre las 19:00 y las 20:00 h. El análisis de trayectorias de retroceso del evento nocturno (Figura 8) identificó la posible intrusión de $\mathrm{O}_{3}$ de la tropósfera libre asociado a un proceso convectivo de plegamiento de la tropopausa. Durante este fenómeno se puede introducir, por periodos cortos de tiempo, $\mathrm{O}_{3}$ de la estratósfera hacia la tropósfera baja, a niveles aún por abajo de los $3000 \mathrm{~m}$ sobre el nivel del mar (Reid y Vaughan 2004, Mavrakis et al. 2010).

Poco después del máximo nocturno, en AMOZ y HUAQ hay una remoción paulatina de $\mathrm{O}_{3}$ dominada por mecanismos de depósito en superficie dados los bajos niveles de NOx en esos sitios, mientras que en CHIP se observa el paso de parcelas de aire provenientes de la ciudad de Puebla con evidencia de titulación al registrarse una anticorrelación entre los $\mathrm{NOx}$ y el $\mathrm{O}_{3}$. Para la medianoche los niveles de $\mathrm{O}_{3}$ se estabilizan en torno al valor de la concentración de fondo en los tres sitios.

\section{1 de abril}

Durante la madrugada los niveles de $\mathrm{O}_{3}$ en $\mathrm{AMOZ}$ fueron relativamente constantes, mientras que en CHIP y HUAQ se observan fuertes variaciones posiblemente asociadas a la presencia de NOx emitidas por fuentes locales cercanas. Durante este periodo y hasta al amanecer dominaron vientos provenientes del norte.

Una vez que amanece hay un rápido incremento en las concentraciones de $\mathrm{O}_{3}$ en los tres sitios en comparación con los dos días previamente analizados. Esto pudo deberse a que este día fue un día inhábil cuando las emisiones matutinas de NOx son menores a las de los días hábiles. En consecuencia, la titulación del $\mathrm{O}_{3}$ por la mañana se reduce y hay mayor acumulación de este contaminante. Destaca en los tres sitios que a pesar de que hay un crecimiento efectivo de las concentraciones de $\mathrm{O}_{3}$ desde el amanecer al mediodía, los picos matutinos de $\mathrm{NO}_{2}$ no superaron las $0.040 \mathrm{ppm}$ y disminuyen rápidamente después de las 09:00 h. El incremento rápido de la concentración de $\mathrm{O}_{3}$ en los tres sitios nuevamente puede explicarse con un acarreo de $\mathrm{O}_{3}$ de arriba de la capa de mezcla a la superficie al desarrollarse ésta durante la mañana.

Los máximos de $\mathrm{O}_{3}$ en CHIP y HUAQ se observaron alrededor de las 12:00 h conforme la proveniencia del viento fue del sector sur-suroeste de acuerdo con la recirculación de masas de aire sobre el VPT detectado con las respectivas TR. El gráfico de las trayectorias de retroceso y avance de la figura 8 muestra claramente este fenómeno. La tendencia de incremento en el $\mathrm{O}_{3}$ continuó por otras $6 \mathrm{~h}$ en AMOZ, mientras que en CHIP y HUAQ se sostuvieron concentraciones elevadas por un periodo de tiempo similar, para después disminuir las 
concentraciones hasta unas $0.050 \mathrm{ppm}$. Después de las 18:00 h se registró el máximo de $\mathrm{O}_{3}$ en $\mathrm{AMOZ}$ en tanto que, en los otros dos sitios, la tendencia de registros constantes de $\mathrm{O}_{3}$ que se mantenía, se descompone y los niveles tienden a reducirse. Esta caída va asociada a un cambio en la dirección del viento ahora proveniente del ESE-E-ENE, situación que se sostiene todavía algunas horas más tarde tal y como lo muestran las trayectorias de la figura 9 con arribo a las 20:00 h de este día.

En los tres días caso analizados se observa una influencia de $\mathrm{O}_{3}$ de la tropósfera libre en algún momento del día que logra mezclarse con parcelas de aire a nivel superficie llevando al incremento en los niveles de este contaminante en los sitios rurales estudiados. Thompson et al. (2008) estudiaron la influencia del $\mathrm{O}_{3}$ en la tropósfera libre sobre la región centro-norte de México con aviones instrumentados durante la campaña MILAGRO de 2006 y propusieron que el $\mathrm{O}_{3}$ en la vertical puede presentar ciertas contribuciones a los niveles de superficie de este contaminante. Sin embargo, no se había confirmado la influencia de este fenómeno en la región centro-sur del país. El análisis que realiza Thompson et al. (2008) del posible origen de este $\mathrm{O}_{3}$, indica que consistentemente proviene del SO, del Océano Pacífico y en ocasiones del Golfo de México, dependiendo de la posición del sistema de alta presión. Los hallazgos de la presente investigación confirman en una buena parte lo propuesto por Thompson et al. (2008) y resaltan la importancia que estos mecanismos de mezclado pueden tener en los niveles de $\mathrm{O}_{3}$ superficiales en cuencas atmosféricas abiertas del centro de México y cuan significante debe ser la consideración del transporte de masas de aire ricas en $\mathrm{O}_{3}$ en las propuestas de estrategias de control de las zonas urbanas de esta región.

\section{CONCLUSIONES}

No obstante que los sitios HUAQ, CHIP y AMOZ se encuentran relativamente cercanos a la zona metropolitana de Puebla, además de estar alineados en las trayectorias de los flujos dominantes de los vientos, no se aprecia que esta área urbana influya notablemente en la acumulación del $\mathrm{O}_{3}$ observado en estos sitios. Sin embargo, se observaron constantes similitudes tanto en los niveles de concentración registrados como en la dinámica de la rapidez en el incremento de $\mathrm{O}_{3}$ medido en los tres sitios que está claramente asociada con la incorporación a la superficie de $\mathrm{O}_{3}$ atrapado por arriba de la capa de inversión. Mientras que la recirculación de masas de aire en el VPT lleva a niveles relativamente altos de $\mathrm{O}_{3}$, las mayores concentraciones de ozono se encuentran ligadas fuertemente con la dominancia de vientos regionales vespertinos del SO combinado con contribuciones de $\mathrm{O}_{3}$ de la troposfera libre acarreados en las parcelas de aire.

La interpretación de los perfiles verticales de $\mathrm{O}_{3}$ para los eventos analizados entre el 17 y 21 de abril con características especiales representativas del tipo de meteorología de la región, confirma que la incorporación de $\mathrm{O}_{3}$ de capas con relativamente alto contenido de este contaminante hacia la superficie al romperse la capa de inversión nocturna y desarrollarse la capa de mezcla, constituye un factor importante en la ocurrencia de niveles altos.

Queda demostrado que las emisiones de precursores de $\mathrm{O}_{3}$ en el VPT no son los únicos responsables de los niveles vespertinos sostenidos de $\mathrm{O}_{3}$ observados en esa región, sino que la combinación entre la formación fotoquímica local, el intercambio entre cuencas, los mecanismos de acarreo de esta especie en la altura, y la influencia sinóptica principalmente de sistemas de alta presión en la recirculación de masas de aire, parecen ser el factor dominante en la persistencia de estos niveles. La implicación de este hallazgo en términos de contaminación por $\mathrm{O}_{3}$ regional y su control, radica en que, si bien hay contribución antrópica a la formación local de $\mathrm{O}_{3}$, las contribuciones externas llevan a la ocurrencia persistente de un mínimo constante de concentración de este contaminante que no sea posible reducir, aún con medidas estrictas que abarquen a la megalópolis del centro de México.

\section{AGRADECIMIENTOS}

Este trabajo forma parte del proyecto "Determinación de tasas de emisión de gases de efecto invernadero de las fuentes de áreas naturales y antropogénicas e identificación de zonas forestales vulnerables del municipio de Puebla y su zona metropolitana al impacto indirecto potencial del cambio climático regional y global" de los Fondos Mixtos del Consejo Nacional de Ciencia y Tecnología de Puebla 77293 (CONACYT/FOMIX-Puebla 77293). Se agradece al Consejo Nacional de Ciencia y Tecnología por el otorgamiento de la beca de posgrado; al Instituto Nacional de Ecología y Cambio Climático (INECC) por el préstamo de su unidad móvil; al Ing. Eduardo Figueroa del Olmo de la empresa TAISA por su apoyo en la preparación de la unidad móvil de la Universi- 
dad Nacional Autónoma de México y a la M. en C. Bertha Eugenia Mar Morales por la generación de mapas con sistema de información geográfica.

\section{REFERENCIAS}

CAMe (2017). Programa de gestión federal para mejorar la calidad del aire de la Megalópolis, PROAIRE 2017-2030. Comisión Ambiental de la Megalópolis [en línea]. https://framework-gb.cdn.gob.mx/data/ institutos/semarnat/Programa_de_Gestión_Federal_2017-2030 final.pdf 12/04/2018

de Foy B., Caetano E., Magaña V., Zitácuaro A., Cárdenas B., Retama A., Ramos A., Molina L.T. y Molina M.J. (2005). Mexico City basin wind circulation during the MCMA-2003 field campaign. Atmos. Chem. Phys. 5 (3), 2503-2558. DOI: 10.5194/acp-5-2267-2005

de Foy B., Varela J.R., Molina L.T. y Molina M.J. (2006). Rapid ventilation of the Mexico City basin and regional fate of the urban plume. Atmos. Chem. Phys. 6, 23212335. DOI: 10.5194/acp-6-2321-2006

de Foy B., Fast J.D., Paech S.J., Phillips D., Walters J.T., Coulter R.L., Martin T.J., Pekour M.S., Shaw W.J., Kastendeuch P.P., Marley N.A., Retama A. y Molina L.T. (2008). Basin-scale wind transport during the MILAGRO field campaign and comparison to climatology using cluster analysis. Atmos. Chem. Phys. 8, 1209-1224. DOI: 10.5194/acp-8-1209-2008

DIAU-BUAP (2008). Clima y urbanización en el Valle de Puebla. Departamento de Investigaciones Arquitectónicas y Urbanísticas, Benemérita Universidad Autónoma de Puebla [en línea]. http://urban.diau.buap. $\mathrm{mx} /$ proyecto.php 29/01/14

Doran J.C., Barnard J.C., Arnott W.P., Cary R., Coulter R., Fast J.D., Kassianov E.I., Kleinman L., Laulainen N.S., Martin T., Paredes-Miranda G., Pekour M.S., Shaw W.J., Smith D.F., Springston S.R. y Yu X-Y. (2007). The T1-T2 study: evolution of aerosol properties downwind of Mexico City. Atmos. Chem. Phys. 7, 1585-1598. DOI: 10.5194/acp-7-1585-2007

Draxler R.R. y Rolph G.D. (2015). HYSPLIT (Hybrid Single-Particle Lagrangian Integrated Trajectory) Model access via NOOA ARL READY [en línea]. https://www.ready.noaa.gov/hypub-bin/trajtype. pl?runtype=archive 19/03/2018

Fast J.D. y Zhong S. (1998). Meteorological factors associated with inhomogeneous ozone concentrations within the Mexico City basin. J. Geophys. Res. 103 (D15), 18927-18946.

Finlayson-Pitts B. y Pitts Jr. J. (2000). Chemistry of the upper and lower atmosphere. Academic Press, San Diego, California, EUA, 969 pp.
INE (2007). Identificación de cuencas atmosféricas en México (INE/A1-011/2007). Informe Final. Instituto Nacional de Ecología, Dirección de Investigación sobre la Calidad del Aire. [en línea]. http://studylib. es/doc/7839422/informe-disponible-en-formato-pdf--instituto-nacional-de 20/03/2018

INE (2009). Comportamiento de los contaminantes en cuencas atmosféricas: metodología y estudio de caso (INE/ A1-004/2009). Informe Final. Instituto Nacional de Ecología [en línea]. http://docplayer.es/13255030-Direccion-general-de-investigacion-sobre-la-contaminacionurbana-y-regional-dgicur-direccion-de-investigacionsobre-la-calidad-del-aire-dica.html 20/03/2018

INE (2011). Diagnóstico sobre compuestos tóxicos en aire ambiente y caracterización espacial de fuentes de emisión y meteorología en la Región Fronteriza de Baja California Norte. (INE/ADE 038/2010). Instituto Nacional de Ecología [en línea]. http://studylib.es/doc/8086623/ estudio-de-caso-de-la-contaminaci\%C3\%B3n-en-lacuenca-atmosf $\%$ C3\%A9rica 20/03/2018

INEGI (2010). Delimitación de las zonas metropolitanas de México 2010. Instituto Nacional de Estadística y Geografia [en línea]. http://www.beta.inegi.org.mx/app/ biblioteca/ficha.html?upc $=702825003884$ 27/03/2018

Jáuregui E.O. (1968). Mesoclima de la región PueblaTlaxcala. Instituto de Geografía, Universidad Nacional Autónoma de México. Ciudad de México, México, 31 pp.

Jazcilevich-Diamant A., García-Reynoso A. y Ruíz-Suárez L.G. (2003). A study of air flow patterns affecting pollutant concentrations in the central region of Mexico. Atmos. Environ. 37 (2), 183-193. DOI: 10.1016/S1352-2310(02)00893-2

Jiménez J., Raga G., Baumgardner D., Castro T., Rosas I., Baez A. y Morton O. (2004). On the composition of airborne particles influenced by emissions of the volcano Popocatépetl in Mexico. Nat. Hazards. 31 (1), 21-37. DOI: 10.1023/B:NHAZ.0000020254.46521.f9

Kley D., Gneiss H. y Volker A.M. (1994). Tropospheric ozone at elevated sites and precursor emissions in the United States and Europe. Atmos. Environ. 28 (1), 149-158. DOI: 10.1016/1352-2310(94)90030-2

Krupa S. (1999). Polución, población y plantas. Montecillo, Colegio de Posgraduados Chapingo. Estado de México, México, 184 p.

Lauer W. y Klaus D. (1975). The thermal circulation of the central Mexican meseta region within influence of the trade winds. Arch. Met. Geoph. Biokl. Ser. B. 23 (4), 343-366. DOI: 10.1007/BF02242690

Lewandowski P.A., Eichinger W. E., Holder H., Prueger J., Wang J. y Kleinman L.I. (2010). Vertical distribution of aerosols in the vicinity of Mexico City during MILAGRO-2006 campaign. Atmos. Chem. Phys. 10, 101-130. DOI: 10.5194/acp-10-1017-2010 
Márquez C., Castro T., Muhlia A., Moya M., MartínezArroyo A. y Báez A. (2005). Measurement of aerosol particles, gases and flux radiation in the Pico de Orizaba National Park, and its relationship to air pollution transport. Atmos. Environ. 39 (21), 3877-3890. DOI: 10.1016/j.atmosenv.2005.03.015

Mavrakis A., Flocas H.A., Mavromatidis E., Kallos G., Theoharatos G. y Christides A. (2010) A case of nighttime high ozone concentration over the greater Athens area. Meteorologische Zeitschrift 19 (1), 35-45.

DOI: 10.1127/0941-2948/2010/0428

Melamed M.L., Basaldud R., Steinbrecher R., Emeis S., Ruíz-Suárez L.G. y Grutter M. (2009). Detection of pollution transport events southeast of Mexico City using ground-based visible spectroscopy measurements of nitrogen dioxide. Atmos. Chem. Phys. 9, 4827-4840. DOI: 10.5194/acp-9-4827-2009

Mena-Carrasco M., Carmichael G.R., Campbell J.E., Zimmerman D., Tang Y., Adhikary B., D'allura A., Molina L.T., Zavala M., García A., Flocke F., Campos T., Weinheimer A.J., Shetter R., Apel E., Montzka D.D., Knapp D.J. y Zheng W. (2009). Assessing the regional impacts of Mexico City emissions on air quality and chemistry. Atmos. Chem. Phys. 9, 3731-3743.

DOI: 10.5194/acp-9-3731-2009

Molina L.T., Kolb C.E., de Foy B., Lamb B.K., Brune W.H., Jimenez J.L. y Molina M. (2007) Air quality in North America's most populous city -overview of the MCMA-2003 campaign. Atmos. Chem. Phys. 7, 2447-2473.

DOI: 10.5194/acp-7-2447-2007

Molina L.T., Madronich S., Gaffney J.S., Apel E., de Foy B., Fast J., Ferrare R., Herndon S., Jimenez J.L., Lamb B., Osornio-Vargas A.R., Russell P., Schauer J.J., Stevens P.S., Volkamer R. y Zavala M. (2010). An overview of the MILAGRO 2006 campaign: Mexico City emissions and their transport and transformation. Atmos. Chem. Phys. 10, 8697-8760.

DOI: $10.5194 /$ acp-10-8697-2010

NREL (2002). Weekend/weekday ozone observations in the South Coast Air Basin-Analysis of air quality data. National Renewable Energy Laboratory. Reporte Final. Reno Nevada, EUA, $32 \mathrm{pp}$.

Padilla V. (2014). Variabilidad temporal de hidrocarburos policíclicos aromáticos (PAHs) en un sitio receptor del Valle Puebla-Tlaxcala: Campaña de monitoreo FOMIX-CONACYT 2012. Tesis de Maestría. Posgrado en Ciencias de la Tierra, Universidad Nacional Autónoma de México. Ciudad de México, México, 108 p.

Reid H.J. y Vaughan G. (2004). Convective mixing in a tropopause fold. Quarterly Journal of the Royal Meteorological Society 130 (599), 1195-1212.

DOI: $10.1256 /$ qj.03.21
Rolph G., Stein A. y Stunder B. (2017). Real-time environmental applications and display system: READY. Environ. Modell. Softw. 95, 210-228.

DOI: $10.1016 /$ j.envsoft.2017.06.025

Ruiz-Suárez L., Torres R., Steinbrecher R., Junkerman W., Torres-Jaramillo A., Mar B. y Hernández-Solís J. (2006). Observations at Tenango del Aire II (chemistry). Memorias. Primera reunión científica de la campaña MILAGRO, Boulder Colorado, EUA. 23 al 25 de octubre, 2006. Cartel.

Ruiz-Suárez L.G., Torres-Jardón R., Jazcilevich-Diamant A., García-Reynoso A., Grutter M., Torres-Jaramillo A., Morales B., Steinbrecher R., Junkermann W. y Molina L. (2009). Analysis of observations and modeling of criteria pollutants and photochemical age indicators during MILAGRO at Tenango del Aire. Memorias. European Geosciences, Union General Assembly, Viena, Austria. 22 al 27 de abril, 2012. CD-ROM.

Ruiz-Suárez L.G., Torres-Jardón R., Woernschimmel H., Steinbrecher R., Junkerman W., Nigenda E., García-Reynoso A., Melamed M., Jazcilevich A., Mar-Morales B., Grutter M. y Molina L. (2010). In depth analysis of the role of the mountain gap south of the Valley of Mexico on the air quality in Mexico City. Memorias. European Geosciences Union, General Assembly, Viena, Austria. 2 de mayo, 2010. CD-ROM.

Salcedo D., Castro T., Ruiz-Suárez L.G., García-Reynoso A., Torres-Jardón R., Torres-Jaramillo J.A., MarMorales B.E., Salcido A., Celada A.T., Carreón-Sierra S., Martínez A.P., Fentanes-Arriaga O.A., Deustúa E., Ramos-Villegas R., Retama-Hernández A., Saavedra M. I. y Suárez-Lastra M. (2012). Study of the regional air quality south of Mexico City (Morelos state). Sci. Total. Environ. 414, 417-432.

DOI: $10.1016 /$ j.scitotenv.2011.09.041

SEDEMA (2018). Secretaría de Medio Ambiente de la Ciudad de México. Sistema de Monitoreo Atmosférico de la Ciudad de México: Base de datos derivados del monitoreo atmosférico por contaminantes para el año 2012 [en línea]. http://www.aire.cdmx.gob.mx/ default.php?opc $=\% 27 \mathrm{aKBhnmI}=\% 27 \&$ opcion $=\mathrm{Zg}==$ 24/04/2018 06/03/2018.

Stein A.F., Draxler R.R., Rolph G.D., Stunder B.J.B., Cohen M.D. y Ngan F. (2015). NOAA's HYSPLIT atmospheric transport and dispersion modeling system, Bull. Amer. Meteor. Soc. 96, 2059-2077.

DOI: 10.1175/BAMS-D-14-00110.1

SSA (2014). Norma Oficial Mexicana NOM-020SSA1-2014. Salud ambiental. Valor límite permisible para la concentración de ozono $\left(\mathrm{O}_{3}\right)$ en el aire ambiente y criterios para su evaluación. Secretaría de 
Salud. Diario Oficial de la Federación. México, 19 de agosto de 2014.

Thompson A.M., Yorks J.E., Miller S.K., Witte J.C., Dougherty K.M., Morris G.A., Baumgardner D., Ladino L. y Rappengluck B. (2008). Tropospheric ozone sources and wave activity over Mexico City and Houston during MILAGRO/Intercontinental Transport Experiment (INTEX-B) Ozonesonde Network Study, 2006 (IONS-06). Atmos. Chem. Phys. 8, 5113-5125. DOI: 10.5194/acp-8-5113-2008
Torres-Jardón R., Reynoso-García A., Jazcilevich A. y Ruíz-Suárez L. (2009). Assessment of the ozonenitrogen oxide-volatile organic compound sensitivity of Mexico City through an indicators-based approach: measurements and numerical simulations comparison. J. Air. Waste. Manag. Assoc. 59 (10),1155-1172. DOI: $10.3155 / 1047-3289.59 .10 .1155$ 